
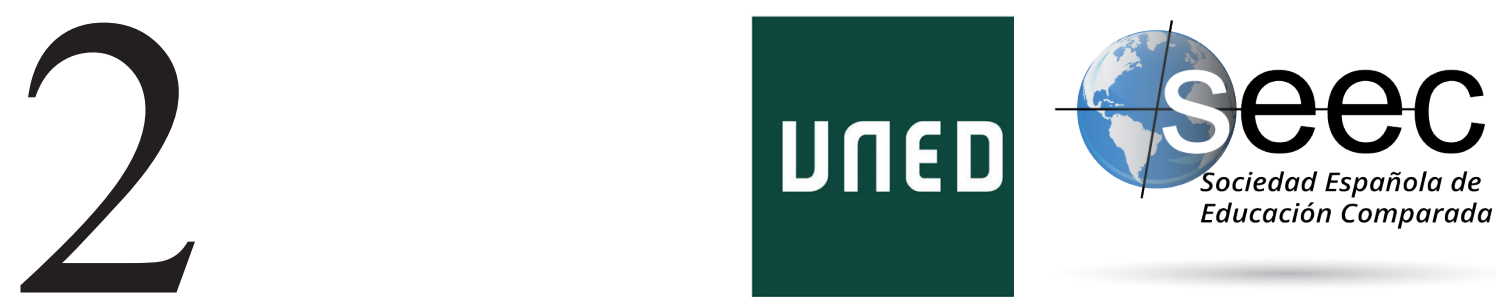

\title{
Los organismos internacionales como sistemas marcadores de tendencias en la Universidad global
}

\author{
International organizations as trend \\ marking systems in the global University
}

\section{Soledad Rappoport*; Bianca Thoilliez ${ }^{* *}$; Tania Alonso-Sainz ${ }^{* * *}$}

DOI: $10.5944 /$ reec.37.2021.27721

\author{
Recibido: 16 de junio de 2020 \\ Aceptado: 31 de agosto de 2020
}

\begin{abstract}
*SolEdAd RAPPOPORT: Profesora Ayudante Doctora (Premio extraordinario, UAM). Departamento de Pedagogía. Universidad Autónoma de Madrid. Magíster en Calidad y Mejora de la Educación (UAM). Licenciada en Pedagogía y Profesora de Educación Primaria (Argentina). Sus principales líneas de investigación son: TIC para la mejora educativa, competencia digital educativa, educación inclusiva, sistemas de apoyo. Pertenece al Grupo de Investigación sobre «Políticas Educativas Supranacionales» (GIPES). Datos de contacto: e-mail: soledad.rappoport@uam.es

**Banca Thoilliez: Profesora Ayudante Doctora en el Departamento de Pedagogía en la Facultad de Formación de Profesorado y Educación de la Universidad Autónoma de Madrid (UAM). Doctora cum laude en Educación con Mención Internacional por la UAM. Becaria del Programa Nacional F.P.U. del Ministerio de Educación, Special Research Student en el Institute of Education University of London, y Visiting Scholar en la Pennsylvania State University. Elected Board Member de la International Network of Philosophers of Education y Visiting Professor de la Faculty of Education Edge Hill University. Su actividad investigadora abarca el estudio de la filosofía y la teoría de la educación, el análisis de políticas educativas y el desarrollo profesional docente. Miembro del Grupo de Investigación sobre «Políticas Educativas Supranacionales» (GIPES) de la UAM, y del Grupo de Investigación de Antropología y Filosofía de la Educación (GIAFE) de la UCM. Datos de contacto: e-mail: bianca.thoilliez@uam.es

***Tania Alonso-Sainz: Doctoranda en Educación (UAM) y Máster en Filosofía de la Educación por el Institute of Education, de la University College London. Licenciada en Pedagogía por la Universidad Complutense de Madrid. Miembro activo del Grupo de Investigación sobre Políticas Educativas Supranacionales (GIPES) y del Grupo de Investigación de Antropología y Filosofía de la Educación (GIAFE-UCM).). Datos de contacto: e-mail: tania.alonso@uam.es
\end{abstract}




\title{
Resumen $^{1}$
}

Distintos autores dan cuenta de una honda transformación de la misión clásica de la Universidad y que en este artículo proponemos identificar con: nuevos paradigmas (aprendizaje a lo largo de la vida y aprendizaje por competencias), nuevas perspectivas (internacionalización y empleabilidad) y nuevos valores (responsabilidad social universitaria y sostenibilidad). Entre las causas de su creciente importancia, identificamos el impulso de distintos organismos y su labor de promoción como sistemas marcadores de tendencias a escala global. A fin de profundizar sobre las características y el alcance de estos cambios, el artículo presenta los resultados de un detallado examen de los documentos publicados en los últimos 10 años por tres organismos internacionales de referencia en el campo de la educación superior: UNESCO, OCDE y OEI. Para alcanzar este objetivo, se llevó a cabo un proceso sistemático de cribado de documentos. Elos materiales seleccionados se indagó sobre la presencia de estas nuevas misiones y el sentido que adquieren en cada organismo estudiado. El artículo aporta, así, evidencias sobre la cuestión y pone a disposición de la comunidad académica una clasificación documental de utilidad para continuar indagando sobre las tendencias globales en el ámbito universitario que promueven con su trabajo los organismos internacionales.

Palabras clave: educación universitaria; educación superior; organismos internacionales; tendencias pedagógicas; misión universitaria.

\begin{abstract}
Different authors report a deep transformation of the classic mission of the University which this article proposes to identify as new paradigms (lifelong learning and learning based competencies), new perspectives (internationalization and employability), and new values (social responsibility and sustainability). Different international organizations account for and promote this trend. In order to delve into the characteristics and scope of the changes, a study has been carried out. It examines documents published in the last 10 years by three international organizations in the field of education: UNESCO, OECD and OEI. To achieve this goal, a systematic document screening process was carried out. The following aprioristic dimensions were identified in the selected materials: lifelong learning, competency-based learning, internationalization, employability, social responsibility and sustainability. In addition, associated emerging themes were sought. The result of the study allows us to provide evidence on the issue and make a useful documentary classification available to continue investigating global trends in the university environment.
\end{abstract}

Keywords: Higher Education; International Organizations; Pedagogical Trends; University Mission. Trabajo desarrollado en el marco del proyecto de I+D+i \#LobbyingTeachers (PID2019-
104566RA-Ioo/AEI/10.13039/501100011033). 


\section{Nuevas misiones de las universidades}

La educación universitaria actual opera en un contexto de grandes desafíos económicos, culturales, políticos y sociales del que no es ajena, por lo que su misión tradicional se está viendo sometida a procesos de cambio (Carrasco, 2017; Esteban, 2019; Hodgson, Vlieghe y Zamojski, 2020; Revesado, 2018; Thoilliez y Valle, 2015). En este escenario de transformaciones profundas, los debates y acciones en el ámbito universitario tienden a enmarcarse en los propios límites de las tendencias propuestas por los organismos internacionales (en adelante, OI) en sus documentos, cuyo estudio detenido se abre a elocuentes interpretaciones sobre la fuerza y eficacia de esta forma de ejercer su influencia política (Manso y Monarca, 2016; Monarca y Manso 2015; Prieto y Manso, 2018; Valle, 2015; Zelaya, 2016). Las recomendaciones que emiten los expertos y técnicos de estos organismos están siendo determinantes en la definición de las agendas nacionales (Thoilliez, 2018), también, a la hora de orientar sus políticas universitarias, las cuales cada vez más entran en frontal oposición con los marcos clásicos de comprensión de la educación universitaria. Así, mientras que tradicionalmente la Universidad ha buscado constituirse y construir espacios apartados del mundo, en los que poder desarrollar prácticas de estudio, legado y generación de conocimientos ${ }^{1}$, las nuevas tendencias en educación superior impulsados por los OI movilizan nuevos paradigmas (aprendizaje a lo largo de la vida y aprendizaje por competencias), perspectivas (internacionalización y empleabilidad) y valores (responsabilidad social y sostenibilidad). Sin menospreciar los beneficios que estas incorporaciones puedan representar para los sistemas de educación superior, creemos importante interrogar los efectos que la imposición/incorporación de estas nuevas misiones universitarias está teniendo ya sobre el sentido, los fines y, por lo tanto, las posibilidades de futuro de la Universidad. Al contrario de lo que se insiste en los documentos de los OI aquí analizados, si se quiere preservar la dimensión educativa de la Universidad habría que mantenerla a cierta distancia de las presiones del mundo económico y social. Algo que pasa por liberarla de la instrumentalización para fines ulteriores a la que, veremos, se encuentra actualmente sometida. A continuación, presentamos brevemente los ejes de las tendencias que hemos identificado en materia de educación superior a cuya generalización global, tal y como trataremos de mostrar después, contribuyen los OI.

\subsection{Nuevos paradigmas: aprendizaje a lo largo de la vida y aprendizaje por competencias}

El lifelong learning o aprendizaje a lo largo de la vida (en adelante, LLL) se articula a partir de dos informes fundamentales de la UNESCO (el Informe Faure, 1972; y, el Informe Delors, 1996). Este paradigma considera al aprendizaje como un proceso sostenido a lo largo de la vida y que, por tanto, no se limita al tiempo de escolarización o matriculación en la educación superior (Espejo, 2006; Medina y Llorent, 2017; Quendler y Lamb, 2016; Valle y Manso, 2017). Los distintos OI han venido concretando en las últimas décadas el papel que desempeña este paradigma en el tratamiento de los problemas educativos mundiales, los desafíos vinculados al desarrollo y el potencial rol que las universidades pueden jugar en él (Eurydice, 2001), concretando las posibilidades del LLL

1 Véanse: Bárcena (2019), Larrosa (2019), Jiménez (2020) y Touriñán (2019). Sobre prácticas de estudio «universitario» llevadas a cabo en los grados de educación, véase Alonso-Sainz y Thoilliez (en prensa). 
en la educación de adultos (OEI, 2010; UNESCO, 2015; OCDE, 2015). En los diferentes sistemas universitarios de todo el mundo, esta nueva manera de entender los ciclos temporales del aprendizaje ha llevado a la puesta en marcha de sistemas alternativos de acceso a la Universidad, modalidades e itinerarios de formación flexibles, sistemas de «universidades populares» o «de mayores», así como a la participación en programas de formación continua (hasta el momento desarrollados mayoritariamente por entidades no universitarias).

Por su parte, el modelo de aprendizaje por competencias (en adelante, $\mathrm{AxC}$ ) es otro gran ejemplo de nuevos paradigmas supranacionales que transita en el ámbito universitario. Sin poder detenernos en las críticas que existen a esta manera de entender el aprendizaje en las universidades (Esteban, 2019; Fulford, 2017; Skea, 2017), puede afirmarse que las competencias se refieren a aprendizajes caracterizados por la integración de conocimientos, destrezas y actitudes (Piñana, 2019; Valle y Manso, 2013). Pensar y planificar las enseñanzas universitarias en línea con el paradigma de AxC, supone combinar las dimensiones: cognitiva (un saber), instrumental (un hacer) y actitudinal (un ser). La generalización y extensión que esta manera de entender los aprendizajes ha encontrado en el contexto universitario da cuenta de cómo la institución universitaria amplía su responsabilidad educadora.

\subsection{Nuevas perspectivas: internacionalización y empleabilidad}

En el actual contexto de globalización, apertura y exposición de la sociedad y economía de los países a un escenario internacional altamente competitivo y cambiante, las universidades (en su triple misión de docencia, investigación y transferencia de conocimiento) enfrentan dos nuevos retos clave: (i) responder a la cada vez mayor demanda de personal cualificado, capaz de innovar y de emprender, que sepa desarrollar su trabajo a nivel global y (ii) atraer talento de estudiantes, docentes e investigadores para aumentar las oportunidades reales de participar en programas, proyectos y redes internacionales.

El nivel de internacionalización al cual las universidades deben aspirar hoy va mucho más allá de la movilidad de estudiantes y la firma de acuerdos bilaterales con otras entidades. Se espera que la internacionalización lo impregne todo: los planes de estudio, los equipos de investigación, titulaciones internacionales conjuntas, campus transnacionales, sistemas internacionalmente acordados de calidad, clasificaciones en los ránquines comparativos, desarrollo de asociaciones de antiguos estudiantes de carácter internacional, pasantías de gestores para el intercambio de buenas prácticas, etc. Un buen ejemplo de esto, son los objetivos estratégicos que el gobierno de España identificó en materia de internacionalización para su sistema educativo universitario para el periodo 2015-2020: Establecer y consolidar un sistema universitario altamente internacionalizado, aumentar el atractivo internacional de las universidades, promover la competitividad internacional del entorno, e intensificar la cooperación en educación superior con otras regiones del mundo (MECD, 2014).

Por su parte, la empleabilidad de los titulados universitarios se ha convertido en un problema social y político de primer orden (Secanella, 2016; Thoilliez, 2014). Es una de las lentes desde las que miramos (y son medidas) las universidades. Aunque, es cierto que las personas con titulación universitaria tienen comparativamente una mejor situación en el momento de buscar, conseguir y mantener en el tiempo un puesto de trabajo, no es menos cierto que el desempleo o la sobrecualificación son hoy por hoy una dramática realidad entre universitarios de muchos países, y de la que no está a salvo ningún sistema 
universitario. Esta realidad ha impulsado la importancia que la Universidad asuma la responsabilidad no solo de formar, sino de asegurar la empleabilidad de sus titulados. Por ello, se ha estrechado el vínculo con las demandas del mercado laboral, la estructura actual de la economía, las relaciones con empresas, y se ha promovido una evaluación del aprendizaje alineado con los marcos de cualificaciones profesionales (Comisión Europea, 2011).

\subsection{Nuevos valores: responsabilidad social y sostenibilidad}

La responsabilidad social universitaria (RSU) tiene su origen en la responsabilidad social empresarial (RSE). La RSE hace alusión al conjunto de obligaciones y de compromisos que una entidad con ánimo de lucro debe asumir como consecuencia del impacto que su actividad económica genera a diferentes niveles (fundamentalmente social y medioambiental). Al ser también las universidades organizaciones que, aún muchas sin ánimo de lucro, pueden impactar de diversos modos sobre la sociedad y las comunidades donde se encuentran ubicadas, en los últimos años estamos asistiendo a cómo la reflexión sobre la responsabilidad social termina llegando igualmente al mundo universitario (MartínezUsarralde, LLoret-Catalá y Mas-Gil, 2017). Se vienen dando diferentes alternativas de actuación a través de las cuales las universidades están tratando de ser coherentes con ese ejercicio de responsabilidad social, como reconoce la UNESCO (2009, p. 2): «la educación superior tiene la responsabilidad social de hacer avanzar nuestra comprensión de problemas polifacéticos con dimensiones sociales, económicas, científicas y culturales, así como nuestra capacidad de hacerles frente». Como han señalado Martínez-Usarralde, LLoret-Catalá y Mas-Gil (2017), la RSU es un área en construcción que se refiere a: (a) que las universidades tienen que aprender de sí mismas desde su gobernanza para el bien de la sociedad, (b) un eje estratégico prioritario, que requiere sistematización y reconocimiento, (c) una tarea integral, reflexiva, académica y ética que se preocupe por los impactos sociales que genera la Universidad en diálogo con otros agentes externos a ella, (d) un compromiso con valores y objetivos sociales que promuevan el desarrollo social, preservando y creando bienes comunes, como la equidad y la calidad de vida de los ciudadanos.

Hoy por hoy, el concepto de sostenibilidad ha ampliado su alcance mucho más de las dimensiones medioambientales y ha pasado a aspirar a mejorar la vida de todos sin dejar a nadie atrás (ONU, 2019), integrándose así en todos los aspectos de la vida de un campus universitario. La sostenibilidad, a partir del impulso de los Objetivos de Desarrollo Sostenible (ONU, 2015), se entiende ya como la vinculación equilibrada de los objetivos de tipo medioambiental, económico y social de las universidades, de un modo tal que se evalúen los efectos a medio y largo plazo de las diferentes actividades (docentes, investigadoras y de transferencia) de un centro universitario (Bautista-Cerro y Díaz, 2017).

La incorporación del valor de la sostenibilidad a la vida universitaria no es algo que haya sucedido de la noche al día, ni debemos asumir acríticamente su aparente neutralidad (Alonso-Sainz, en prensa). Es el resultado de un progresivo acuerdo internacional (Alba, 2017) para el fomento de la sostenibilidad en la Universidad. Por su parte, de acuerdo con las directrices que las universidades españolas se han dado a sí mismas a través del órgano de coordinación de rectores CRUE (2012), la consideración del valor de la sostenibilidad en las universidades debería asumir los siguientes objetivos: (1) comprender la interacción de la Universidad con el ambiente exterior, (2) entender la contribución económica, política, cultural y social de la Universidad, (3) trabajar interdisciplinariamente para promover, con enfoque holístico, cambios sostenibles, (4) 
participar en las propuestas sociales de desarrollo sostenible, (5) aplicar las innovaciones profesionales a la protección de los derechos humanos, (6) incluir la voz de la ciudadanía para que tengan parte en su propio desarrollo.

A fin de explorar el alcance de estos nuevos paradigmas, valores y perspectivas, en este artículo nos proponemos examinar su presencia en los documentos producidos por tres OI de referencia que funcionan como sistemas marcadores de tendencia en la educación superior a escala global: la Organización para la Cooperación y el Desarrollo Económicos (en adelante, OCDE), la Organización de las Naciones Unidas para la Educación, la Ciencia y la Cultura (en adelante, UNESCO) y la Organización de Estados Iberoamericanos (en adelante, OEI).

\section{Metodología}

Para alcanzar el objetivo planteado se propuso un estudio de tipo exploratorio que indagase, a través de un proceso de cribado, la presencia las tendencias apriorísticas y presentadas en el marco teórico, en los documentos elaborados a propósito de la educación superior por la UNESCO, OCDE y OEI, durante los últimos 10 años (2010-2019).

Para la selección de los tres organismos se tuvieron en cuenta los siguientes criterios: a) referencia del organismo en el ámbito educativo mundial; b) diversidad de contextos de influencia y enfoques asumidos; c) disponibilidad de documentos en las respectivas páginas web institucionales; d) posibilidades y conveniencia del equipo de investigación.

Para el diseño metodológico se plantearon estrategias de recogida de información comunes para los tres organismos y otras adaptadas a los diferentes sistemas de gestión de conocimiento con que trabajan sus archivos documentales digitales (sitio web y/o bibliotecas en línea). En los tres organismos, la exploración se ha hecho a través de los correspondientes sitios web y bibliotecas digitales. En todos los casos se ha seguido un proceso de selección sistemática de documentos, guiado por criterios de exclusión previamente acordados. Este proceso se desarrolló en las siguientes etapas:

- Fase 1. Identificación: en cada sitio repositorio digital se identificaron los documentos publicados durante el período 2010-2019 referidos a la educación superior.

- Fase 2. Cribado: se depuraron las bases de datos creadas a partir de la fase identificación, eliminando duplicaciones y documentos que no respondían por fechas o temática.

- Fase 3. Elegibilidad: se excluyeron los documentos que, aunque archivados por el OI, su autoría no pertenecía al organismo correspondiente y/o miembro/s del equipo directivo.

- Fase 4. Definición: a partir de los documentos elegibles, para cada organismo se procedió a definir la muestra final atendiendo a las particularidades de cada caso indagado.

A partir de este diseño común y atendiendo a las singularidades de cada organismo, sitio web, materiales elaborados y formatos de publicación, se han realizado adaptaciones metodológicas, siendo las más significativas las correspondientes a la OEI. Las definiciones de la conformación de las muestras para los tres casos se detallan en el Anexo 1. 
En el siguiente gráfico se presenta la síntesis del proceso de reducción de documentos.

\begin{tabular}{|c|c|c|c|c|c|c|c|}
\hline : & $\begin{array}{c}\text { UNESCO } 912 \\
\text { OCDE } 238 \\
\text { OEI } 172\end{array}$ & ט. & $\begin{array}{c}\text { UNESCO } 883 \\
\text { OCDE } 193 \\
\text { OEI } 45\end{array}$ & 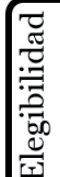 & $\begin{array}{c}\text { UNESCO } 133 \\
\text { OCDE } 188 \\
\text { OEI } 35\end{array}$ & 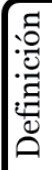 & $\begin{array}{l}\text { UNESCO } 80 \\
\text { OCDE } 26 \\
\text { OEI } 33\end{array}$ \\
\hline
\end{tabular}

Figura 1. Cantidad de documentos elegidos en cada fase del proceso de conformación de la muestra documental para los tres organismos.

En los documentos seleccionados de cada organismo se examinó la presencia (o ausencia) de seis ámbitos de interés, definidos en el marco teórico centrado en esas «nuevas misiones» de las universidades:

Tabla 1.

Esferas y ámbitos de exploración

\begin{tabular}{ll}
\hline ESFERAS & ÁMBITOS \\
\hline \multirow{2}{*}{ Paradigmas } & Aprendizaje a lo largo de la vida [LLL] \\
\cline { 2 - 2 } & Aprendizaje por competencias [AxC] \\
\hline \multirow{2}{*}{ Perspectivas } & Internacionalización [INT] \\
\cline { 2 - 2 } & Empleabilidad [EMP] \\
\hline \multirow{2}{*}{ Valores } & Responsabilidad social universitaria [RSU] \\
\cline { 2 - 2 } & Sostenibilidad [SOST] \\
\hline
\end{tabular}

Una vez etiquetados los documentos en función de los temas apriorísticos, se completó la indagación con un análisis de tipo inductivo. A fin de determinar el sentido que adquieren las temáticas en los OI estudiados, en cada grupo de materiales se realizaron distintas lecturas de sus elementos más significativos para entender la toma general de posición temática, prestando atención prioritaria a los abstracts y resúmenes ejecutivos. Cuando estos no se encontraban en los documentos, se analizaban la introducción y otros fragmentos destacados. Este proceso permitió identificar elementos recurrentes para las temáticas, según cada caso. Por último, se llevó a cabo un nuevo proceso de etiquetamiento de documentos. El software Excel ha sido la principal herramienta de apoyo durante el proceso descrito. Los documentos se han ordenado en tres bases de datos distintas, cuyas clasificaciones se han ido ajustando a medida que se profundizaban las lecturas y sus consecuentes identificaciones temáticas.

Se presentan a continuación los resultados para cada uno de los OI cuya documentación en materia de educación superior ha sido sometida a análisis. 


\section{Resultados}

\subsection{La UNESCO por una educación superior al servicio de un proyecto de mundo más sostenible y equitativo}

\subsubsection{La educación superior en UNESCO}

La UNESCO ${ }^{2}$ es la única entidad especializada de las Naciones Unidas que cuenta con atribuciones en materia de educación superior, facilitando la preparación de políticas en materia de enseñanza superior. De acuerdo con la meta 4.3 del Objetivo de Desarrollo Sostenible 4: «Para 2030, asegurar el acceso en condiciones de igualdad para todos los hombres y las mujeres a una formación técnica, profesional y superior de calidad, incluida la enseñanza universitaria», la UNESCO ofrece soporte técnico a los Estados Miembros para que puedan evaluar sus estrategias y políticas relativas a la educación superior para mejorar el acceso en condiciones de equidad a una educación superior de calidad. La educación superior es, también, un elemento esencial para lograr otros objetivos: fin de la pobreza (ODS 1); salud y bienestar (ODS 3); igualdad de género (ODS 5); trabajo decente y crecimiento económico (ODS 8); producción y consumo responsables (ODS 12); acción por el clima (ODS 13); y paz, justicia e instituciones sólidas (ODS 16). El Marco de Acción Educación 2030 (UNESCO, 2016), que concreta el trabajo a desarrollar para la implementación del ODS4, se asienta sobre dos pilares políticos fundamentales: (i) favorecer las oportunidades de aprendizaje, (ii) reducir las barreras para que nadie quede excluido de dichas oportunidades. El Marco de Acción insta a desarrollar acuerdos internacionales en el ámbito de la educación superior de manera que se garanticen sistemas adecuadamente establecidos y normalizados, ampliando el acceso, la igualdad, la calidad y la pertinencia de sus enseñanzas. También busca mitigar la diferencia entre el contenido de las enseñanzas impartidas y lo que el alumnado debe aprender para asegurar el desarrollo sostenible y un buen aprovechamiento de las tecnologías, los recursos educativos abiertos y la enseñanza a distancia. La UNESCO, también brinda apoyo técnico y elabora instrumentos jurídicos para fortalecer y dinamizar los sistemas de educación superior en todo el mundo. Para ello, lleva estos últimos años preparando el que será el «Convenio Mundial sobre Reconocimiento de Cualificaciones relativas a la Educación Superior». Además, la UNESCO ha establecido alianzas con centros de educación superior con su programa UNITWIN y Cátedras UNESCO, el cual, tras 25 años en funcionamiento, anima la cooperación y el establecimiento de redes entre universidades para fortalecer sus capacidades de intercambio de conocimientos y de trabajar en común.

A fin de mostrar una primera aproximación a la importancia que la UNESCO concede al ámbito de trabajo de la educación superior, se expone la proporción de documentos elaborados que abordan la temática universitaria frente al total de sus publicaciones y al material dedicado a la educación no universitaria.

Del total de 46.754 registros documentales que ha producido la UNESCO durante el periodo 2010-2019, 8.272 corresponden a la temática 'educación' y 883 a la de 'educación superior'. La educación superior representa un 8,82 \% sobre el total de producción documental sobre educación y un 1,5 \% sobre el total de producción documental global.

2 Para un estudio más completo sobre los diferentes ámbitos de trabajo de la UNESCO y su relevancia en el campo de la educación, véase Martínez-Usarralde, Viana Orta y Villarroel (2015). 


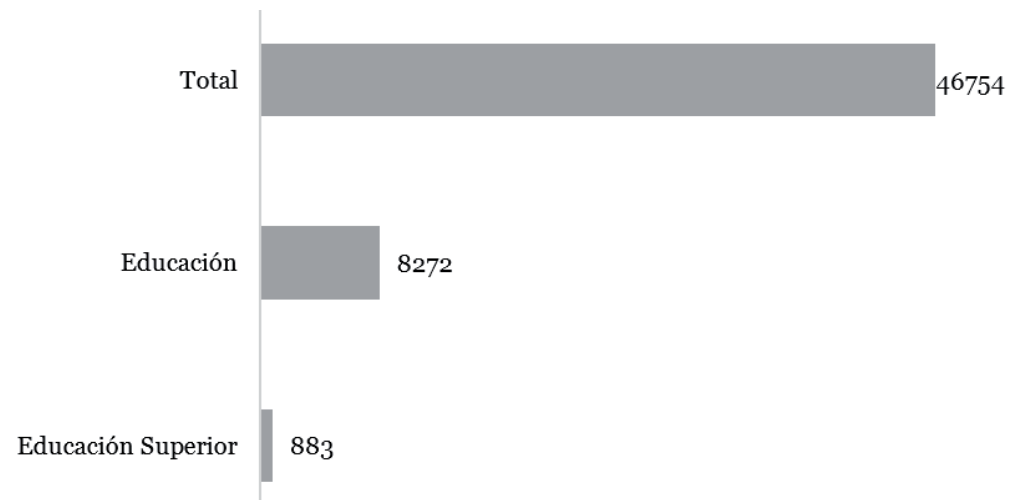

Figura 2. Publicaciones totales, documentos sobre educación y sobre educación superior de UNESCO, periodo 2010-2019.

\subsubsection{Las definiciones temáticas en la UNESCO}

A partir de los 80 documentos incluidos finalmente en el análisis ${ }^{3}$, y tomando como referencia la descripción de las esferas y los ámbitos definidos desde el marco teórico, emergieron 17 temas que catalizan el trabajo de la UNESCO en materia de educación superior y que resumimos en la Tabla 2.

Tabla 2.

Ámbitos, temas y documentos UNESCO, periodo 2010-2019

\begin{tabular}{|c|c|c|}
\hline \multirow{4}{*}{$\begin{array}{l}\text { ÁMBITOS } \\
\text { [LLL] }\end{array}$} & TEMAS & DOCUMENTOS \\
\hline & Modalidades e itinerarios (MOD.IT) & $\begin{array}{l}\mathrm{UNo}_{0}, \mathrm{UN}_{17}, \mathrm{UN}_{43}, \mathrm{UN}_{45}, \mathrm{UN}_{6}, \mathrm{UN}_{71} \\
\mathrm{UN}_{76}, \mathrm{UN}_{77}, \mathrm{UN}_{80}\end{array}$ \\
\hline & Educación de adultos (ED ADUL) & UNo9, UN $16, \mathrm{UN}_{17}, \mathrm{UN}_{27}, \mathrm{UN}_{74}$ \\
\hline & Formación continua (FORM.CONT) & $\mathrm{UN}_{12}, \mathrm{UN}_{45}, \mathrm{UN}_{66}, \mathrm{UN}_{74}$ \\
\hline$[\mathrm{AxC}]$ & $\begin{array}{l}\text { Competencias del siggo XXI } \\
\text { (COMP.SXXI) }\end{array}$ & $\begin{array}{l}\mathrm{UN}_{22} \text {, UN27, UN64, UN66, UN67, UN6s } \\
\text { UN UN80 }\end{array}$ \\
\hline \multirow[t]{4}{*}{ [INT] } & Educación a distancia (ED.DIST) & UN14, UN64, UN68 \\
\hline & Reconocimiento de títulos (REC.TíT) & $\begin{array}{l}\mathrm{UN}_{10}, \mathrm{UN}_{15}, \mathrm{UN}_{19}, \mathrm{UN}_{20}, \mathrm{UN}_{36}, \mathrm{UN}_{37} \\
\mathrm{UN}_{44}, \mathrm{UN}_{46} 6, \mathrm{UN}_{47} 7, \mathrm{UN}_{4} 8, \mathrm{UN}_{49}, \\
\mathrm{UN}_{50}, \mathrm{UN}_{51}, \mathrm{UN}_{52} \text {, UN } 53, \mathrm{UN}_{54}, \mathrm{UN}_{60} \\
\text { UN61, UN64, UN } 74\end{array}$ \\
\hline & ProgramaUNITWIN (UNITWIN) & $\mathrm{UNO}_{4}, \mathrm{UN}_{26} 6, \mathrm{UN}_{59}, \mathrm{UN} 78$ \\
\hline & Movilidad (MOV) & $\mathrm{UN}_{41}, \mathrm{UN}_{44}, \mathrm{UN}_{4}, \mathrm{UN}_{75}, \mathrm{UN}_{79}$ \\
\hline \multirow[t]{2}{*}{ [EMP] } & $\begin{array}{l}\text { Empleabilidad factor de calidad } \\
\text { (IND.CAL) }\end{array}$ & $\mathrm{UN}_{3} 8, \mathrm{UN}_{4} \mathrm{O}, \mathrm{UN}_{55}, \mathrm{UN}_{57}, \mathrm{UN}_{71}, \mathrm{UN}_{72}$ \\
\hline & Mercadolaboral (MER.LAB) & $\begin{array}{l}\text { UNo8, UNo9, UN } 11, \mathrm{UN}_{13}, \mathrm{UN}_{33}, \mathrm{UN}_{34} \\
\mathrm{UN}_{3} 8, \mathrm{UN}_{44}, \mathrm{UN}_{57}, \mathrm{UN}_{62}, \mathrm{UN}_{70}, \mathrm{UN}_{71} \\
\text { UN8。 }\end{array}$ \\
\hline \multirow[t]{3}{*}{ [RSU] } & Gobernanza responsable (GOB.RESP) & 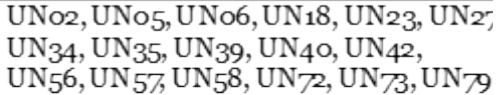 \\
\hline & Conocimiento distribuido (CON.DISTR) & UN29, UN30, UN66 \\
\hline & Responsabilidad financiera (RESP.FIN) & $\mathrm{UN}_{41}, \mathrm{UN} 62, \mathrm{UN} 63, \mathrm{UN}_{70}, \mathrm{UN}_{75}$ \\
\hline \multirow[t]{4}{*}{ [SOST] } & Gobernanza sostenible (GOB.SOST) & $\mathrm{UNo}_{3}, \mathrm{UN}_{14}, \mathrm{UN}_{16}, \mathrm{UN}_{24}, \mathrm{UN}_{29}$ \\
\hline & Sostenibilidad curricular (SOST.CURR) & UNo1, UNo7, UN59, UN67, UN69 \\
\hline & Desarrollo sostenible (DES.SOST) & $\mathrm{UN}_{11}, \mathrm{UN}_{12}, \mathrm{UN}_{13}, \mathrm{UN}_{16}, \mathrm{UN}_{21}, \mathrm{UN}_{22}$ \\
\hline & Equidad (EQUI) & $\begin{array}{l}\mathrm{UN}_{2}, \text { UNo } 8, \mathrm{UN}_{13}, \mathrm{UN}_{16}, \mathrm{UN}_{18} 8, \mathrm{UN}_{2} 5 \\
\mathrm{UN}_{2} 8, \mathrm{UN}_{29}, \mathrm{UN}_{31}, \mathrm{UN}_{32}, \mathrm{UN}_{43}, \mathrm{UN}_{4} 5 \\
\mathrm{UN}_{3}, \mathrm{UN}_{74}\end{array}$ \\
\hline
\end{tabular}

3 El detalle del procedimiento de cribado seguido para el caso de la UNESCO se encuentra explicado en el Anexo 1, apartado A. El listado completo de los 80 documentos sometidos a análisis, de los que la Tabla 2 recoge su clave de clasificación, se encuentran en el Anexo 2. 
Las menciones sobre el aprendizaje a lo largo de la vida [LLL] se vinculan principalmente a tres temas: a) diversidad de modalidades e itinerarios (MOD.IT) en el acceso y el tránsito por estudios de educación superior; b) la atención específica que requiere la planificación y asistencia a la educación de adultos (ED.ADUL); y c) el papel de los programas de formación continua (FORM.CONT) en el contexto de la educación superior.

Empezando por el primero de estos temas (MOD.IT), la UNESCO cuenta entre sus preocupaciones las de mejorar las vías de comunicación entre la Formación Profesional y la Universidad, apuntándose al problema del reconocimiento de las titulaciones de Formación Profesional por parte de las instituciones universitarias, así como a la necesidad de pensar en formas de mejorar el atractivo social de la Formación Profesional. También, la UNESCO promueve y propone ideas para diversificar las vías de acceso a la Universidad y favorecer diferentes modalidades formativas de educación superior. En sus documentos se encuentran argumentos que explican cómo la diversificación en esta materia ha afectado a todos los aspectos de la educación superior. Las razones que justifican esta aparentemente necesaria diversificación son variadas: por un lado, la masificación de las universidades que empuja hacia un cambio de modelo, y, por otro lado, el crecimiento de la demanda social por modalidades de educación superior alternativas a la oferta universitaria tradicional. El segundo de los temas (ED.ADUL), se justifica como un aspecto definitorio de la filosofía educativa de la UNESCO y que debe poner en marcha la búsqueda de recursos y enfoques que hagan accesible la educación superior a la población adulta. Ofrecer educación de nivel universitario a todos, en todas las etapas de la vida, es, para la UNESCO una misión fundamental de estas instituciones. En el tercer tema de trabajo dentro del ámbito del aprendizaje a lo largo de la vida (FORM.CONT), la idea fundamental es que un titulado universitario no queda convertido, automáticamente en un profesional, hay más formación por adquirir en el desarrollo de la vida laboral. La formación continua es presentada, también, desde los documentos de la UNESCO, como la mejor respuesta a las cambiantes demandas profesionales del mercado laboral.

Con respecto a las menciones sobre el aprendizaje por competencias $[\mathrm{AxC}]$ se ha concretado una única temática central: el papel que se le asigna a la educación superior para desarrollar las competencias del siglo XXI (COMP.SXXI) entre estudiantes y profesionales. Esta temática se manifiesta en dos vertientes en los documentos analizados. Por un lado, se insiste en la necesidad de repensar el currículo, ofreciéndose como razones para este replanteamiento urgente que las barreras entre asignaturas y materias deben derribarse, o que este giro es necesario para que las nuevas generaciones estén convenientemente orientadas hacia el futuro. Por otro lado, pero relacionado con este último punto, se discute la relación de oposición entre una formación enfocada en los contenidos y una formación enfocada en el desarrollo de competencias.

Por su parte, las menciones sobre la internacionalización [INT] se han manifestado en cuatro temáticas prevalentes: a) el potencial de internacionalización que representa para universidades y otras entidades de educación superior ofrecer programas formativos de educación a distancia (ED.DIST); b) la importancia de promover un reconocimiento garantista de los títulos (REC.TÍT) entre regiones y países; c) el programa UNITWIN como sistema de colaboración directo con y sobre las universidades; y d) la promoción de la movilidad (MOV) entre estudiantes y profesorado universitario. Empezando por la primera de estas cuatro temáticas (ED.DIST), la UNESCO entiende muy claramente que la educación a distancia es un medio idóneo para internacionalizar las audiencias universitarias, siendo una alternativa que se añade, sin suplantar, a la oferta de enseñanzas 
presenciales que permite internacionalizar las instituciones y rentabilizar su oferta académica. En este contexto, la aparición de instituciones universitarias totalmente online hace replantear la idea de una Universidad que educa a las élites de cada país, caminándose hacia una idea de Campus de masas global. Pasando al segundo tema (REC.TÍT), el estudio documental realizado y el análisis sobre su contenido desarrollado, ponen de manifiesto los intensos trabajos preparatorios para la firma de acuerdos regionales para el reconocimiento de titulaciones entre países. La UNESCO defiende activamente los beneficios de una progresiva armonización educativa, acompañada de una confianza mutua entre países que permita el reconocimiento de las cualificaciones que unos y otro emiten. Dentro del tercer tema (UNITWIN), el análisis de contenido arroja el papel estratégico que la UNESCO reconoce a estas cátedras para colaborar de manera más directa con las instituciones universitarias. Por último, en el cuarto tema (MOV), la UNESCO defiende una movilidad en educación superior que cruce fronteras y que contribuya a la cooperación y el mejor entendimiento entre países. Unas políticas de movilidad que acentúan la competición entre instituciones. Esto hace entrar a las universidades en la dinámica de maximizar su internacionalización por los beneficios en términos reputacionales y contables que reporta.

Pasando al ámbito de la empleabilidad [EMP], la UNESCO lo trabaja en torno a dos grandes ejes temáticos: a) el cuidado de las tasas de empleabilidad de los titulados como factor de calidad (IND.CAL) de las instituciones de educación superior; y b) la capacidad y la necesidad de responder y adaptarse a las necesidades del mercado laboral (MER.LAB) que se espera de universidades y otras entidades de educación superior. Así, sobre el primero de estos dos temas (IND.CAL), la idea central es que los sistemas de garantía de calidad se están utilizando no solo para optimizar los procesos de enseñanzaaprendizaje, sino también para mejorar los índices de empleabilidad de las titulaciones que ofertan, siendo un aspecto que ha ganado progresiva importancia, también por el peso relativo que tiene en la configuración de los rankings de calidad universitaria. El segundo tema presente en el ámbito de la empleabilidad (MER.LAB), aborda la urgencia de ir adaptando las cualificaciones profesionales, incluidas las que se adquieren en los niveles de educación superior, a un mercado laboral cambiante. También se manifiesta la preocupación por la falta específica de profesionales de la rama de carreras STEM y la educación de ingenieros, considerados puestos de trabajo altamente demandados y necesarios para el desarrollo de los países, así como de ajustar mejor la oferta de formación con las necesidades del mercado laboral.

La responsabilidad social universitaria [RSU] también queda confirmada como uno de los ámbitos de trabajo de la UNESCO en materia de educación superior, concretándose en tres ejes temáticos: a) la promoción de una gobernanza responsable (GOB. RESP) por parte de las instituciones proveedores de educación superior; b) la inserción de política de conocimiento distribuido (CON.DISTR) por parte de las universidades; y c) favorecer estrategias de responsabilidad financiera (RESP.FIN) en la gestión de sus recursos. Empezando por el primer tema de los tres (GOB.RESP), destacan la preocupación por eliminar las prácticas de corrupción universitaria, la gestión propiamente dicha, así como el diseño y desarrollo de política de profesorado universitario. A la UNESCO le preocupa especialmente que en una oferta de educación superior cada vez más globalizada, la oferta académica en todos los países cumpla unos estándares adecuados de calidad, siendo la supervisión de estos una muestra de ejercicio de responsabilidad por parte de los gobiernos. Y ello especialmente en un contexto de progresiva incorporación 
de agente privados en la provisión de enseñanzas de nivel universitario. También trabaja la dimensión social de esa gestión universitaria, desde el señalamiento de la necesidad de responder a su contrato social, hasta el estudio del impacto de los rankings de calidad universitarios. La segunda temática de trabajo que emergió dentro del ámbito de la responsabilidad social universitaria ha sido el del conocimiento distribuido (CON.DISTR) y la tercera, la responsabilidad financiera (RESP.FIN), entendiendo por tal, por un lado, el ajuste de la relación coste-beneficio en las inversiones en educación superior, y, por otro lado, la financiación público-privado de la educación terciaria.

Por último, la promoción de los valores de sostenibilidad [SOST] se concretan, a su vez, en cuatro ejes temáticos de trabajo: a) promover mecanismos que aseguren una gobernanza sostenible (GOB.SOST) de las instituciones; b) introducir elementos que aseguren la sostenibilidad curricular (SOST.CURR) en las actividades de enseñanza; c) promover que las instituciones se preocupen y ocupen por contribuir de manera activa al desarrollo sostenible (DES.SOST); y d) asegurar políticas que garanticen la equidad (EQUI) en su acceso y funcionamiento interno. La primera temática (GOB.SOST) se traduce en recomendaciones para que las universidades, dentro de sus marcos de garantía de calidad, introduzcan elementos que evalúen el impacto que tienen sobre el desarrollo de los territorios donde se implantan, así como la recomendación de diferentes esquemas para asegurar su sostenibilidad económica. Por su parte, el tema de la sostenibilidad curricular (SOST.CURR) se plantea en términos de gran reto para las universidades, presentándose como un contenido sobre el que las instituciones tienen la obligación de alfabetizar a sus estudiantes. En lo que respecta a la tercera temática vinculada al ámbito de la sostenibilidad (DES.SOST), la UNESCO se muestra, también en este punto, especialmente preocupada por la medida en que las soluciones para los retos futuros exigen contar con ingenieros y profesionales del ámbito STEM que estén bien formados. Finalmente, la temática de la equidad (EQUI) resulta clave para comprender cómo la UNESCO plantea su visión sobre la sostenibilidad. Una de las ideas centrales que atraviesa varios documentos es que un mundo marcado por las desigualdades no es ni puede ser nunca sostenible. Resulta, por lo tanto, fundamental reducir al mínimo y tratar de eliminar todas las barreras de acceso a la educación terciaria, presentando especial atención a los sistemas de admisión y cuidando de manera especial a grupos tradicionalmente excluidos de los estudios superiores.

\subsubsection{La prevalencia de los ámbitos y sus temas en la UNESCO}

El proceso analítico seguido ha hecho posible cuantificar los resultados obtenidos. Así, atendiendo a los ámbitos de trabajo, se observa (ver Figura 3) que en el caso de la UNESCO destacan dos: la internacionalización (24 \%) y la sostenibilidad (23 \%). Seguidamente, presta atención a la responsabilidad social universitaria (17\%), a la empleabilidad (14 \%) y al lifelong learning (14\%). En último lugar (6 \%) se encuentra el aprendizaje por competencias (6\%). 


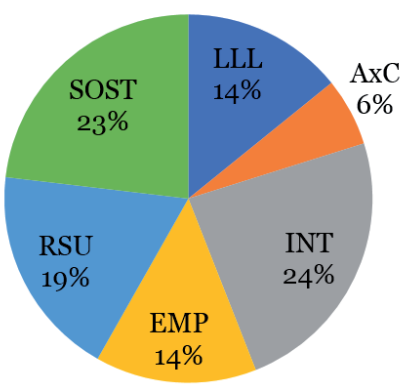

Figura 3. Ámbitos abordados en los documentos UNESCO, periodo 2010-2019

En cuanto a la evolución de estos ámbitos en la última década (ver Figura 4), podemos destacar dos datos: la producción de documentos ha sido irregular, sin responder a un patrón definido, apreciándose un pico en el año 2017. La atención a los diferentes temas responde también a un patrón irregular.

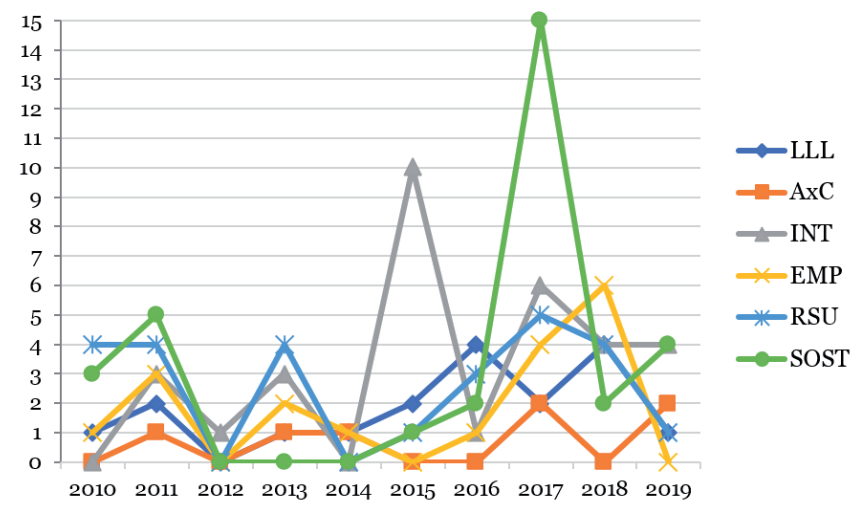

Figura 4. Evolución de ámbitos abordados en documentos UNESCO, periodo 2010-2019

Tal y como se refleja en la figura 5, osl temas más trabajados en los documentos sobre educación superior analizados de la UNESCO a lo largo de esta última década han sido el reconocimiento de títulos (dentro del ámbito [INT]), la gobernanza responsable (dentro del ámbito [RSU]) y el desarrollo sostenible (incluido en el ámbito [SOST]).

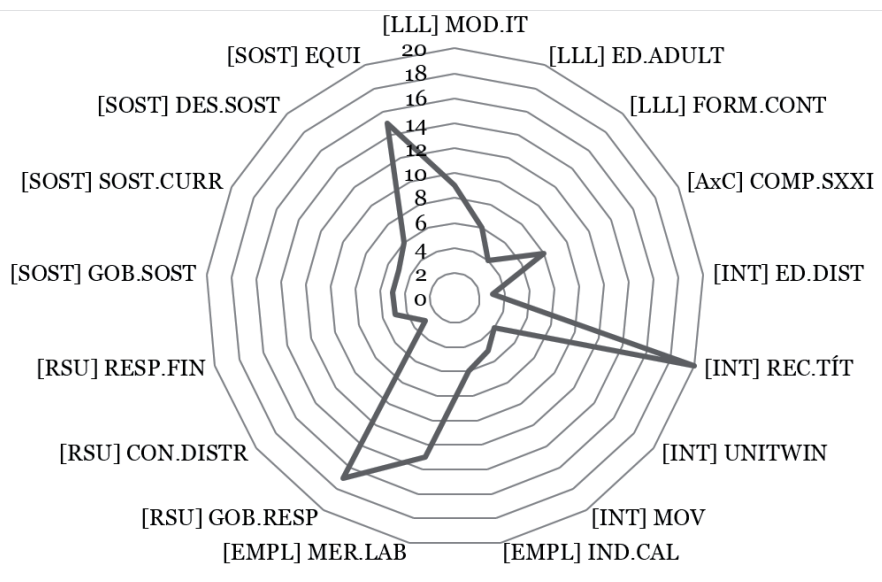

Figura 5. Cantidad de documentos UNESCO según temas abordados, periodo 2010-2019 


\subsection{OCDE, la importancia de la equidad para asegurar la RSU}

\subsubsection{La educación universitaria en la OCDE}

La OCDE cuenta con un grupo de profesionales dedicado exclusivamente al análisis y producción de política educativa universitaria. Este equipo, según cuenta el propio organismo (OCDE, 2020), lleva a cabo análisis sobre una amplia gama de sistemas y políticas de educación superior. Para ello, cuentan con el asesoramiento del Grupo de Trabajo Informal sobre educación superior (IWGHE, en sus siglas en inglés), que se reúne regularmente para revisar y comentar sus actividades, y cuyos delegados generalmente incluyen representantes responsables de la política de educación superior dentro de los ministerios de educación u otros organismos gubernamentales, expertos e invitados del área. La actividad de la OCDE en materia de educación superior se concreta en seis elementos, que incluyen acciones, programas y equipos de trabajo.

En primer lugar, encontramos el proyecto Labour Market Relevance and Outcomes of Higher Education que proporciona recomendaciones prácticas sobre cómo las políticas de educación superior de cada país miembro de la OCDE pueden promover en los titulados universitarios una buena inserción e impacto en el mercado laboral. En segundo lugar, ofrecen The Higher Education Resources Project que pretende ayudar a los policy-makers a organizar los presupuestos y la asignación de recursos, ofreciendo talleres en los que los expertos de los países pueden revisar mutuamente las medidas adoptadas y compartir experiencias. Además, mediante las National Reviews of Higher Educación Policy la OCDE ofrece revisiones personalizadas del marco de gobernanza, o de alguna faceta particular de la política superior, a demanda de los propios países, para ayudar así a los desafíos concretos que enfrentan. Por otro lado, llevan a cabo lo que el propio organismo llama Enhancing the Higher Education Evidence Base, que se basa en el apoyo a la expansión de la política basada en evidencias de educación superior. A partir de encuestas periódicas pretenden aportar datos a los países para mejorar el análisis de sus políticas y orientar la toma de decisiones. En quinto lugar, The Informal Working Group on Higher Education (IWGHE) es un grupo de trabajo, como ya se ha mencionado, que desarrolla y supervisa los proyectos centrados en sistemas y políticas de educación superior, y que trabaja periódica y estrechamente con los profesionales de la OCDE. Por último, la acción más conocida de este, y otros organismos, son sus publicaciones, que ofrecen revisiones sistemáticas y recomendaciones generales en educación superior para ayudar a los investigadores y responsables políticos a guiar su acción. Una aportación esencial a este respecto es la OECD iLibrary que ofrece al gran pública una biblioteca virtual para encontrar cada una de sus publicaciones, ordenadas por temática, año, país, autoría y tipo de documento.

En la figura 6 se puede observar que la OCDE entre 2010 y 2019 dedicó un 11,75 \% del total de su producción al área de educación. Si nos detenemos en la atención concreta que se ha prestado desde la OCDE a la educación universitaria entre 2010 y 2019, observamos que un 7,1\% de los documentos dedicados a educación se centraban en el estudio, análisis y recomendaciones sobre educación superior. Este porcentaje no alcanza al $1 \%$ cuando nos fijamos en el peso de los informes de educación superior dentro del total de la producción de la OCDE. 


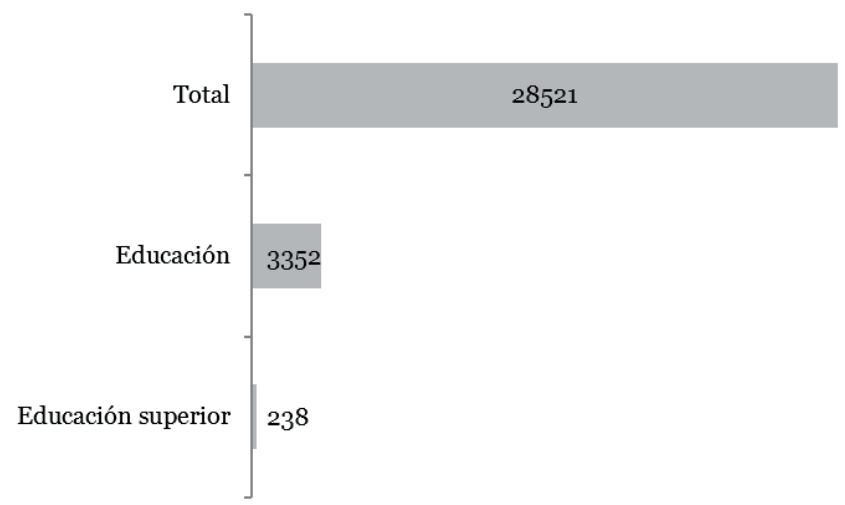

Figura 6. Publicaciones totales, documentos sobre educación y sobre educación superior de la OCDE, periodo 2010-2019.

\subsubsection{Las definiciones temáticas en la OCDE}

A partir del proceso de etiquetado y clasificación de los 26 documentos que resultaron tras el cribado de las 238 iniciales $^{4}$, se obtuvieron un total de 13 temas (resumidos en la Tabla 3):

Tabla 3.

Ámbitos, temas y documentos OCDE, periodo 2010-2019

\begin{tabular}{|c|c|c|}
\hline ÁMBITOS & TEMAS & DOCUMENTOS \\
\hline \multirow{2}{*}{ [LLL] } & Modalidades e itinerarios (MOD.IT) & $\mathrm{OC} 8, \mathrm{OC} 14, \mathrm{OC} 17$ \\
\hline & Innovación (INNOV) & OC16 \\
\hline \multirow[t]{2}{*}[\mathrm{AxC}]{} & Competencias S.XXI (COMP.S.XXI) & $\mathrm{OC} 13$ \\
\hline & Metodología docente (MET.DOC) & $\mathrm{OC}_{14}, \mathrm{OC} 26$ \\
\hline \multirow[t]{3}{*}{ [INT] } & Movilidad de estudiantes (MOV.EST) & OCo1, OCo3 \\
\hline & Colaboración internacional (COL.INT) & OC10, OC16, OC17 \\
\hline & Armonización de titulaciones (ARM.TIT) & $\mathrm{OCo} 1, \mathrm{OC} 22, \mathrm{OCo} 7, \mathrm{OC}_{17}, \mathrm{OC}_{21}$ \\
\hline \multirow[t]{2}{*}{ [EMP] } & Mercado laboral (MER.LAB) & $\mathrm{OCo}_{3}, \mathrm{OC}_{8}, \mathrm{OC}_{15}$ \\
\hline & $\begin{array}{l}\text { Inversión y coste de oportunidad } \\
\text { (INV.CO) }\end{array}$ & OC18, OC19 \\
\hline \multirow[t]{2}{*}{ [RSU] } & Equidad (EQUI) & OCo1, OCo5, OCo6, OC12, OC14, OC17 \\
\hline & Financiación (FIN) & OCo1, OC11, OC24 \\
\hline \multirow[t]{2}{*}[\mathrm{SOST}]{} & Sostenibilidad financiera (SOST.FIN) & OCo1, OCo2, OC2O, OC22 \\
\hline & Gobernanza (GOB) & OCo9, OC11, OC17, OC20, OC25 \\
\hline
\end{tabular}

Para la OCDE, el paradigma del aprendizaje permanente [LLL] se vincula principalmente a dos realidades: a) modalidades e itinerarios (MOD.IT) y b) innovación (INNOV). En primer lugar, se entiende que el Lifelong Learning tiene que ver con la necesidad de flexibilizar las modalidades e itinerarios (MOD.IT) de los estudiantes del siglo XXI, con el fin de promover su acceso. Asimismo, se asocia el aprendizaje a lo largo de toda la

4 El detalle del procedimiento de cribado seguido para el caso de la OCDE se encuentra explicado en el Anexo 1, apartado B. El listado completo de los 13 documentos sometidos a análisis, de los que la Tabla 3 se recoge su clave de clasificación, se encuentran en el Anexo 3. 
vida con la capacidad de innovación (INNOV) que tiene la Universidad y la necesidad de transformación de roles de la institución, así como de los docentes y de los estudiantes. De esta manera, los cambios permitirán el aprendizaje sostenible.

Por su parte, el paradigma de aprendizaje por competencias [AxC] se expresa en los documentos de la OCDE mayoritariamente mediante dos temáticas: a) el impulso e implementación de las competencias del Siglo XXI (COMP.SXXI), y b) las metodologías docentes (MET.DOC). El primero insiste en la importancia de formar y evaluar en las competencias que demanda el siglo XXI (COMP.S.XXI), en una economía basada en el conocimiento. Por su parte, el segundo de los aspectos del aprendizaje por competencias $[\mathrm{AxC}]$ se refiere a las innovaciones didácticas (MET.DOC) que han de emprender los docentes de educación superior, que puede resumirse en una metodología más competencial y aplicada, más centrada en la resolución de problemas y, por tanto, menos centrada en las clásicas tareas universitarias de estudio y formación disciplinar.

En cuanto a la perspectiva de internacionalización [INT] que adopta la OCDE, se puede formular que son tres los temas que la conforman: a) movilidad de estudiantes (MOV.EST), b) colaboración internacional (COL.INT) y c) armonización de titulaciones (ARM.TIT). La movilidad de estudiantes a nivel internacional (MOV.EST) es un elemento recurrente en los documentos de la OCDE y se refiere a potenciar en intercambio y la movilidad de estudiantes entre las distintas universidades para fomentar ciudadanos y trabajadores globales. Por su parte, la colaboración internacional (COL.INT) entre instituciones internacionales transfronterizas de educación superior es, según la OCDE, clave para el desarrollo de la mejora de la educación superior en cada uno de los países miembro. Además, es nuclear la importancia de la armonización (ARM.TIT) en las titulaciones y en las condiciones de acceso a la educación universitaria entre países para que pueda darse una completa internacionalización. En general, la internacionalización es un elemento transversal a todas las propuestas de la OCDE en materia de educación superior, siendo los temas mencionados los más sobresalientes.

Con relación a la perspectiva de la empleabilidad [EMP], la OCDE fija su atención en dos temas: a) mercado laboral (MER.LAB) y b) inversión y coste de oportunidad (INV. CO). El primero se refiere a la vinculación entre la Universidad y el mercado laboral (MER.LAB), pero además del mercado laboral, y este es el segundo elemento, se refieren a menudo a la inversión y coste de oportunidad (INV.CO) de los estudiantes con el fin de adquirir un trabajo con perspectivas económicas positivas. Por lo tanto, la empleabilidad en los documentos analizados de la OCDE guarda estrecha relación con la necesidad de preparar a los estudiantes universitarios para el mercado laboral (MER.LAB) actual y sus necesidades, y reflexionar sobre la inversión que hacen los estudiantes y el coste de oportunidad (INV.CO) que les supone; reflexión que desemboca en reforzar la perspectiva de la empleabilidad como una responsabilidad de la Universidad actual.

En cuanto a los valores de la educación universitaria que transitan por los documentos de la OCDE, se observan cuatro. Dentro de la responsabilidad social universitaria [RSU], la OCDE identifica como claves para la RSU los siguientes temas: a) la equidad (EQUI) y b) las posibilidades de financiación (FIN) de los estudiantes socioeconómicamente más desfavorecidos. Sobre el primero, la OCDE insiste en la importancia de atender, mediante las políticas universitarias, a los estudiantes más vulnerables. Y destacan que asegurar la financiación de los grados universitarios a los estudiantes es uno de los principales retos a los que se enfrenta, hoy día, la Universidad en el marco de la RSU. 
El valor de la sostenibilidad [SOST] está compuesto en el marco de la OCDE por dos principales temas: a) sostenibilidad financiera (SOST.FIN) y b) gobernanza (GOB). Consideran que el futuro de la Universidad pasa por la sostenibilidad financiera (SOST. FIN), entendida esta como la capacidad de organizar y combinar presupuestos públicos y privados, para prever un futuro universitario económicamente sostenible. Además, insisten en el elemento de la gobernanza (GOB), entendida como la capacidad de gestión, organización y liderazgo ante los distintos desafíos actuales, como un aspecto clave para el mantenimiento de los objetivos universitarios a largo plazo.

\subsubsection{La prevalencia de los ámbitos y sus temas en la OCDE}

Atendiendo a los parámetros, se observa (ver Figura 4) que dentro de los temas que ha abordado la OCDE en materia de educación superior destacan dos: la responsabilidad social (25\%) y la sostenibilidad (24\%). Seguidamente, ha prestado atención a la internacionalización (17\%), empleabilidad (15\%), aprendizaje por competencia (12 \%) y lifelong learning (7\%).

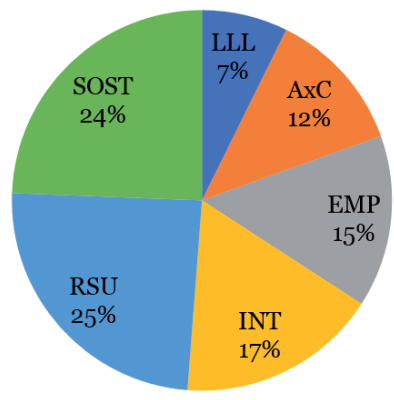

Figura 7. Ámbitos abordados en los documentos OCDE, periodo 2010-2019

En cuanto a la evolución de los parámetros en la última década (ver Figura 8), podemos destacar dos datos: el número de producción de documentos ha sido irregular, sin responder a ningún patrón, y es significativo el aumento de documentos sobre RSU en el año 2013.

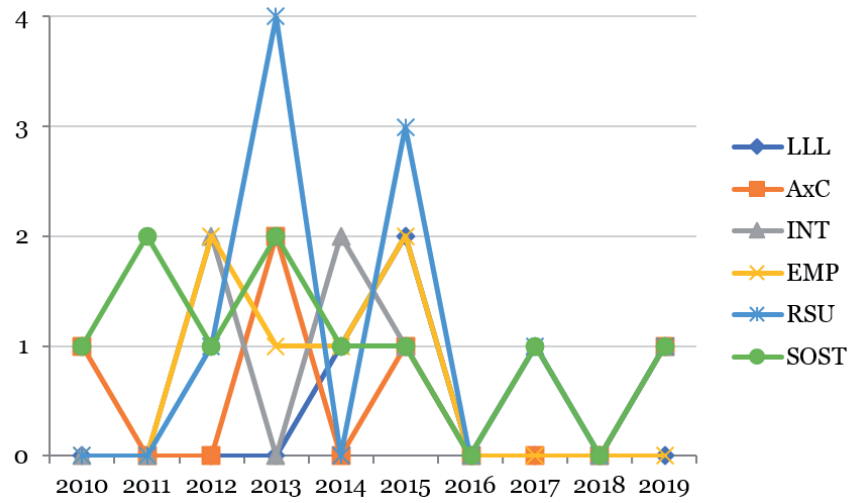

Figura 8. Evolución de ámbitos abordados en documentos OCDE, periodo 2010-2019.

El indicador más trabajado en los documentos cribados de la OCDE (ver Anexo 3) en la última década fue, con diferencia, la equidad, es decir, la posibilidad de que todos los jóvenes accedan a la Universidad sin estar limitados por sus condiciones de partida. 
También tuvieron mucho protagonismo la armonización internacional de los títulos y de otros elementos de las universidades, y la gobernanza, entendida como la capacidad de liderar las instituciones de educación superior, priorizar los retos, y aprovechar los recursos.

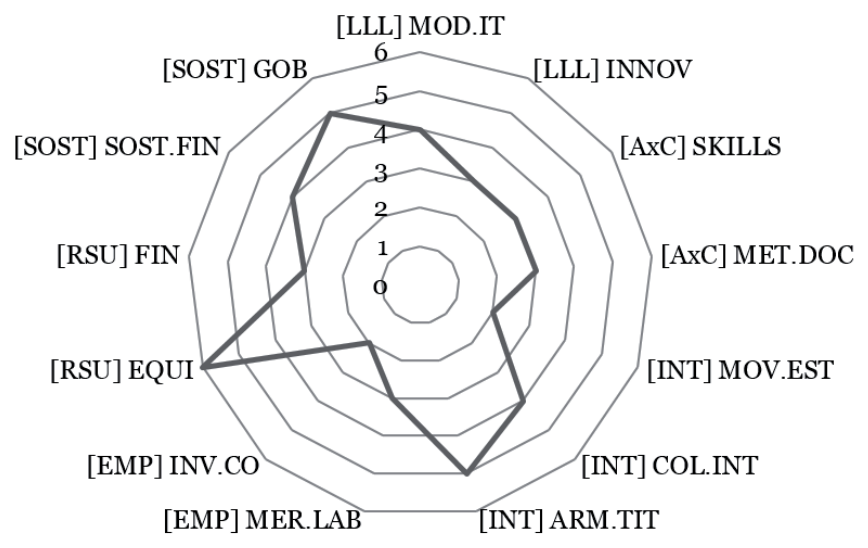

Figura 9. Cantidad de documentos OCDE según temas abordados, periodo 2010-2019

\subsection{OEI, la cooperación como herramienta transformadora}

\subsubsection{La educación universitaria en la OEI}

La OEI reconoce la educación universitaria como el ámbito clave para avanzar hacia la integración, cooperación y el desarrollo regional. Los objetivos planteados para esta etapa buscan generar espacios compartidos, lograr acuerdos de transparencia y asegurar la calidad en todos los sistemas educativos, así como potenciar la misión investigadora que posibilite la prosperidad de los países de la región. Dan muestra de ello, el interés por consolidar el Espacio Iberoamericano de Conocimiento, el desarrollo de la estrategia Universidad Iberoamericana 2030 y la estrecha vinculación que se plantea entre universidad, ciencias y tecnología.

El cribado realizado permite observar la prevalencia de la educación superior dentro de la OEI. En el período 2010-2019, de todos los trabajos referidos al campo educativo, casi el 20 \% tratan sobre la educación superior de manera parcial o exclusiva (ver Figura 10).

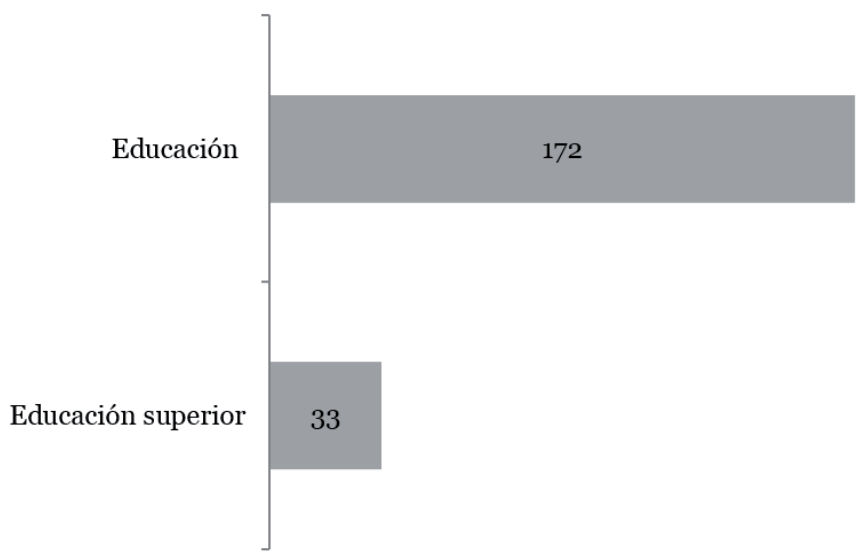

Figura 10. Documentos OEI sobre educación y sobre educación universitaria, periodo 2010-2019 
Por otra parte, hay ciertos indicadores cualitativos que permiten ver la atención del organismo hacia la educación superior. Esta etapa adquiere un tratamiento distinto al resto del campo educativo. En la página web, por ejemplo, para el área educativa se distinguen dos pestañas: a) educación y b) educación superior y ciencia. Esta vinculación entre la universidad y el campo científico se evidencia también en la existencia del Observatorio de Ciencia, Tecnología y Sociedad.

Por último, en el documento Metas Educativas 2021 (OEI, 2010), una de las once metas generales, la novena, se dedica exclusivamente al ámbito universitario y la conformación del Espacio Iberoamericano de Conocimiento.

\section{3•3.2. Las definiciones temáticas en la OEI}

A través de la exploración sobre los ámbitos realizada en los 33 documentos seleccionados5 se identificaron las siguientes temáticas asociadas y que se resumen en la Tabla 4:

Tabla 4.

Ámbitos, temas y documentos OEI, periodo 2010-2019

\begin{tabular}{|c|c|c|}
\hline ÁMBITOS & TEMAS & DOCUMENTOS \\
\hline \multirow[t]{2}{*}{ [LLL] } & $\begin{array}{l}\text { Derecho a la educación } \\
\text { (DER.EDU) }\end{array}$ & $\begin{array}{l}\text { OE01, OE02, OE04, OE05, OEo6, Eo7, } \\
\text { OE10, OE12, OE13, OE16, OE19, OE20, } \\
\text { OE21, OE22, OE26 }\end{array}$ \\
\hline & Articulación (ART) & $\begin{array}{l}\text { OE02, OE04, OE05, OE06, OE13, E19, } \\
\text { OE20, OE25, OE26 }\end{array}$ \\
\hline \multirow[t]{2}{*}[\mathrm{AxC}]{} & $\begin{array}{l}\text { Propósito educativo } \\
\text { (PROP.EDU) }\end{array}$ & $\begin{array}{l}\text { OE02, OE03, OE04, OE05, OE06, E07, } \\
\text { OE08, OE12, OE13, OE16, OE17, OE18, } \\
\text { OE19, OE20, OE21, OE22, OE25, OE26, } \\
\text { OE29, OE30 }\end{array}$ \\
\hline & $\begin{array}{l}\text { Validación y acreditación } \\
\text { (VAL.ACRED) }\end{array}$ & $\begin{array}{l}\text { OE02, OEo4, OE05, OE06, OEo7, E13, } \\
\text { OE19, OE26, OE30 }\end{array}$ \\
\hline \multirow[t]{3}{*}[\mathrm{INT}]{} & Movilidad (MOV) & $\begin{array}{l}\text { OE01, OE02, OE03, OE04, OE05, Eo6, } \\
\text { OE07, OE08, OE09, OE10, OE11, OE12, } \\
\text { OE13, OE16, OE17, OE18, OE19, OE20, } \\
\text { OE21, OE22, OE26, OE28, OE29, E30, } \\
\text { OE31 }\end{array}$ \\
\hline & $\begin{array}{l}\text { Colaboración y coperación } \\
\text { (COL.COOP) }\end{array}$ & $\begin{array}{l}\text { OE01, OE02, OE03, OE04, OE05, Eo6, } \\
\text { OE07, OE08, OE09, OE10, OE11, OE12, } \\
\text { OE13, OE14, OE16, OE17, OE18, OE19, } \\
\text { OE20, OE21, OE22, OE24, OE26, E28, } \\
\text { OE29, OE30, OE31 }\end{array}$ \\
\hline & Armonización (ARMON) & $\begin{array}{l}\text { OE01, OE02, OE03, OE04, OEo5, Eo6, } \\
\text { OE07, OE08, OE09, OE12, OE13, OE14, } \\
\text { OE15, OE18, OE19, OE20, OE21, OE22, } \\
\text { OE26, OE28, OE29, OE30, OE31 }\end{array}$ \\
\hline \multirow[t]{2}{*}{ [EMP] } & $\begin{array}{l}\text { Educación Técnico Profesional } \\
\text { (ETP) }\end{array}$ & $\begin{array}{l}\text { OE02, OE04, OE05, OE06, OE07, } \\
\text { OE12, OE13, OE16, OE18, OE19, OE20, } \\
\text { OE21, OE22, OE26, OE30 }\end{array}$ \\
\hline & Apertura curricular (AP.CURR) & $\begin{array}{l}\text { OE02, OE04, OEo5, OEo6, OEo7, Eo8, } \\
\text { OE13, OE19, OE20, OE21, OE22, OE26, } \\
\text { OE28 }\end{array}$ \\
\hline \multirow[t]{2}{*}{ [RSU] } & Equidad e Inclusión (EQUI.INC) & $\begin{array}{l}\text { OE02, OE03, OE04, OEo5, OEo6, Eo8, } \\
\text { OE12, OE13, OE15, OE18, OE19, OE20, } \\
\text { OE21, OE22, OE23, OE26, OE27, E28, } \\
\text { OE30, OE32 }\end{array}$ \\
\hline & $\begin{array}{l}\text { Vinculación con el entorno } \\
\text { (VIN.ENT) }\end{array}$ & $\begin{array}{l}\text { OE01, OE02, OE03, OE4, OE05, OEo6, } \\
\text { OE08, OE11, OE12, OE13, OE18, OE19, } \\
\text { OE24, OE26, OE27, OE29, OE } 31, \text { OE } 32\end{array}$ \\
\hline \multirow[t]{2}{*}{ [SOST] } & Expansión (EXP) & $\begin{array}{l}\text { OE01, OE02, OE03, OE04, OEo6, Eo8, } \\
\text { OE11, OE13, OE18, OE19, OE20, OE21, } \\
\text { OE22, OE23, OE24, OE26, OE27, E28, } \\
\text { OE32 }\end{array}$ \\
\hline & $\begin{array}{l}\text { Financiación y gobernanza } \\
\text { (FIN.GOB) }\end{array}$ & $\begin{array}{l}\text { OE02, OE03, OEo4, OE06, OEo8, OE11, } \\
\text { OE13, OE19, OE20, OE22, OE23, OE25, } \\
\text { OE27, OE28, OE31 }\end{array}$ \\
\hline
\end{tabular}

$5 \quad$ El detalle del procedimiento y las especiales particularidades del cribado seguido para el caso de la OEI se encuentra explicado en el Anexo 1, apartado C. El listado completo de los 33 documentos sometidos a análisis, de los que la Tabla 4 recoge su clave de clasificación, se encuentran en el Anexo 4. 
Las menciones sobre el aprendizaje a lo largo de la vida [LLL] se vinculan principalmente a dos temas: a) el derecho a la educación de todas las personas (DER.EDU) para participar en forma plena en la sociedad; y b) la articulación (ART) entre etapas educativas y entre ámbitos de aprendizaje para permitir la formación continua. Las referencias encontradas en los documentos relacionan el aprendizaje a lo largo de la vida a procesos de educación de adulto, principalmente alfabetización y educación superior técnico profesional. La educación universitaria, sin embargo, está prácticamente ausente en las explicitaciones de los elementos que componen este tema.

La presencia del paradigma competencial [COMP] se entiende como propósito educativo (PROP.EDU). Para la OEI, esta misión es imprescindible y adquiere un sentido doble: individual y social. Por un lado, la educación por competencias se vincula al desarrollo de habilidades, conocimientos y actitudes necesarias para que cada persona pueda participar activamente en todos los ámbitos de la vida, haciendo especial alusión al ámbito laboral. Por otra parte, formar ciudadanos competentes es necesario para que Iberoamérica pueda avanzar hacia el desarrollo regional. El enfoque, además, trae aparejado la necesaria validación y acreditación de las cualificaciones y competencias vinculadas a la demanda laboral.

Como es de esperar para un organismo de cooperación internacional, en los documentos analizados, la internacionalización [INT] es el ámbito vinculado a la educación universitaria más mencionada. El principal objetivo de internacionalización es la construcción de un Espacio Iberoamericano de Conocimiento (EIC). Este fin actúa como brújula y orienta los distintos elementos estratégicos que componen la internacionalización: a) movilidad (MOV); b) cooperación y colaboración (COL.COOP); y c) armonización (ARMON). Los tres elementos se interrelacionan y son atravesados por un factor transversal: la calidad educativa. La OEI plantea movilidades de estudiantes, profesores e investigadores como principal estrategia de internacionalización. A través de las movilidades (MOV), se busca el intercambio de buenas prácticas, la conformación de redes universitarias y la creación de lazos de cooperación y colaboración entre investigadores de distintos países para la producción conjunta. Asimismo, la colaboración y cooperación (COL.COOP) entre agencias se observa en distintas iniciativas de formación interuniversitarias llevadas a cabo por la OEI, a través del Centro de Altos Estudios Universitarios (CAU). Avanzar hacia la construcción del EIC, requiere, además, de la armonización de títulos (ARMON). Este desafío, pone de relevancia otras dos necesidades: contar con un sistema de aseguramiento de la calidad de títulos y disponer de indicadores nacionales fiables, que posibiliten la comparabilidad y garanticen el seguimiento de la calidad.

La empleabilidad [EMP] se asocia principalmente a dos temáticas: a) la educación técnico profesional (ETP) y b) la apertura curricular al mercado laboral (AP.CURR). Dentro de la educación superior, la ETP se posiciona como la estrategia clave para atender una de las mayores urgencias de la región: la inserción laboral. Para ello, se busca mejorar la concordancia entre el mercado laboral y la formación y proponer planes de estudios acordes a las competencias demandadas por el ámbito laboral. Cabe señalar que si bien la vinculación empleabilidad-universidad está presente en algunos documentos de la OEI (en especial a partir del 2015), el desarrollo de la empleabilidad de los ciudadanos aparece mayormente ligada a la educación técnico profesional.

El ámbito responsabilidad social universitaria [RSU] se expresa en dos cuestiones: a) la vinculación con el entorno (VIN.ENT) y b) la participación de mujeres en carreras 
tradicionalmente asociadas al género masculino, como las ciencias, tecnología, ingenierías y matemáticas (STEM). La primera, aborda el rol de la Universidad en la sociedad. Contempla las tradicionales acciones de extensión universitaria y la apertura institucional al contexto para co-producir, junto a otros sectores, conocimiento que atienda a la demanda local y al desarrollo regional. Bajo esta temática se destaca la preocupación por avanzar hacia sistemas universitarios más equitativos en cuanto a la presencia de alumnado procedente de grupos sociales minoritarios, como afroamericanos y pueblos originarios.

Respecto a la sostenibilidad universitaria [SOST], los documentos analizados, plantean dos temas principales: a) las estrategias, problemáticas y características en la consolidación y expansión de la matriculación de estudiantes en educación universitaria (EXP); y b) la financiación y gobernanza de las instituciones (FIN.GOB). En cuanto a la expansión de la matrícula, se destaca el avanza de la modalidad virtual, la diversificación institucional y el aumento de la oferta universitaria privada. Esta expansión trae aparejada como problemática la acreditación de títulos y el control de la calidad. Por eso, la temática de la sostenibilidad es atravesada por dos factores recurrentes ya mencionados: la necesidad de contar con un sistema de aseguramiento de la calidad, así como de avanzar hacia la construcción de indicadores y datos nacionales fiables y comparables para el seguimiento de la calidad. La financiación y la gobernanza son otros elementos que definen la sostenibilidad en la OEI. Los documentos analizados dan cuenta de la atención puesta en la inversión pública y privada para el desarrollo de proyectos $\mathrm{I}+\mathrm{d}$, financiar becas y hacer posible la oferta educativa universitaria en general. Asimismo, se examina el tipo de gestión de la oferta universitaria y las condiciones laborales de la plantilla docente investigadora en las universidades de la región.

\section{3•3•3. La prevalencia de los ámbitos y sus temas en la OEI}

Al contabilizar los documentos según los ámbitos abordados se observa una marcada presencia de la internacionalización [INT]. Asimismo, se distingue el aprendizaje a lo largo de la vida [LLL] como el paradigma menos contemplado.

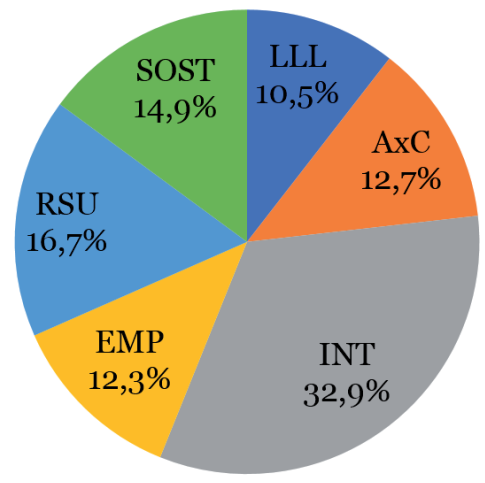

Figura 11. Ámbitos abordados en documentos OEI, periodo 2010-2019

En cuanto a la evolución a lo largo de los años se observa que la cantidad de publicaciones sobre educación universitarias no ha seguido una trayectoria regular durante el período estudiado. En cuanto a sus ámbitos, como es de esperar, en la última década prevalecen documentos que tratan la internacionalización [INT], siendo especialmente notoria la presencia de esta cuestión respecto al resto, en el año 2014. 


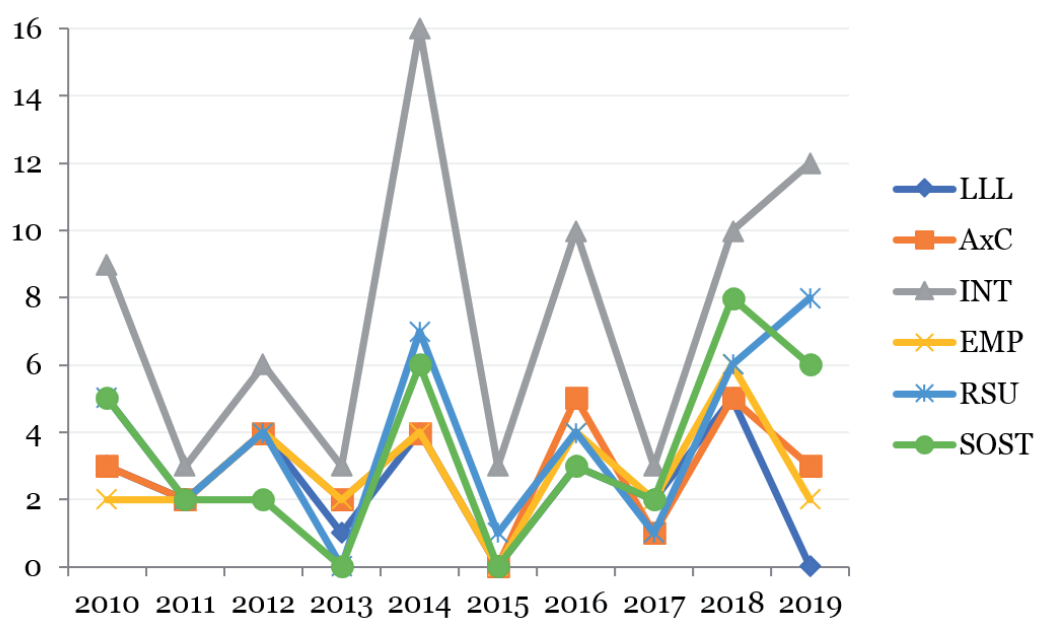

Figura 12. Evolución de ámbitos abordados en documentos OEI, periodo 2010-2019

$\mathrm{Al}$ indagar sobre los elementos que componen cada uno de los temas, se observa una marcada presencia de la colaboración y cooperación (COL.COOP) con 27 documentos que lo abordan, seguido por el sub-tema movilidad (MOV), con 25 materiales de referencia. Por el contrario, el elemento articulación (ART), referido a la necesidad de mejorar las transiciones entre etapas y la vinculación entre educación formal e informal para asegurar el aprendizaje a lo largo de la vida [LLL] y la validación y acreditación (VAL. ACRE) del aprendizaje por competencias [AxC] son los elementos menos abordados en los documentos sobre educación universitaria.

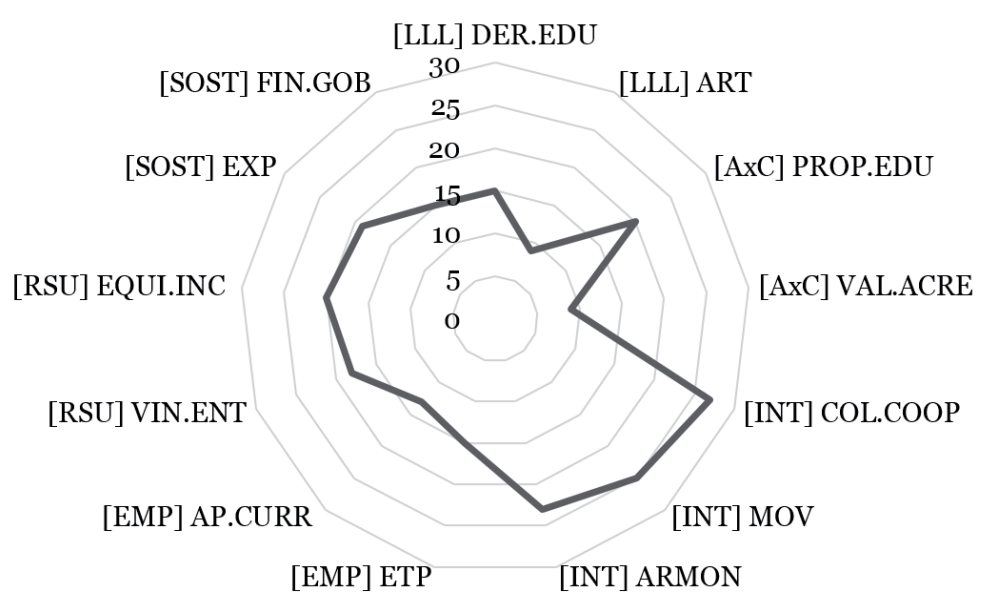

Figura 13. Cantidad de documentos OEI según temas abordados, periodo 2010-2019

\section{Discusión y conclusiones}

Este artículo ha puesto de relieve que asistimos, desde hace unas décadas, a una honda transformación de la misión clásica de la Universidad. A su vez, hemos comprobado que son los distintos OI los que impulsan la incorporación de tres esferas, que resultan en seis ámbitos: (A) nuevos paradigmas (1-LifelongLearning y 2-aprendizaje por competencias), (B) nuevos valores (3-internacionalización y 4-empleabilidad) y (C) nuevas perspectivas (5-responsabilidad social universitaria y 6-sostenibilidad). 


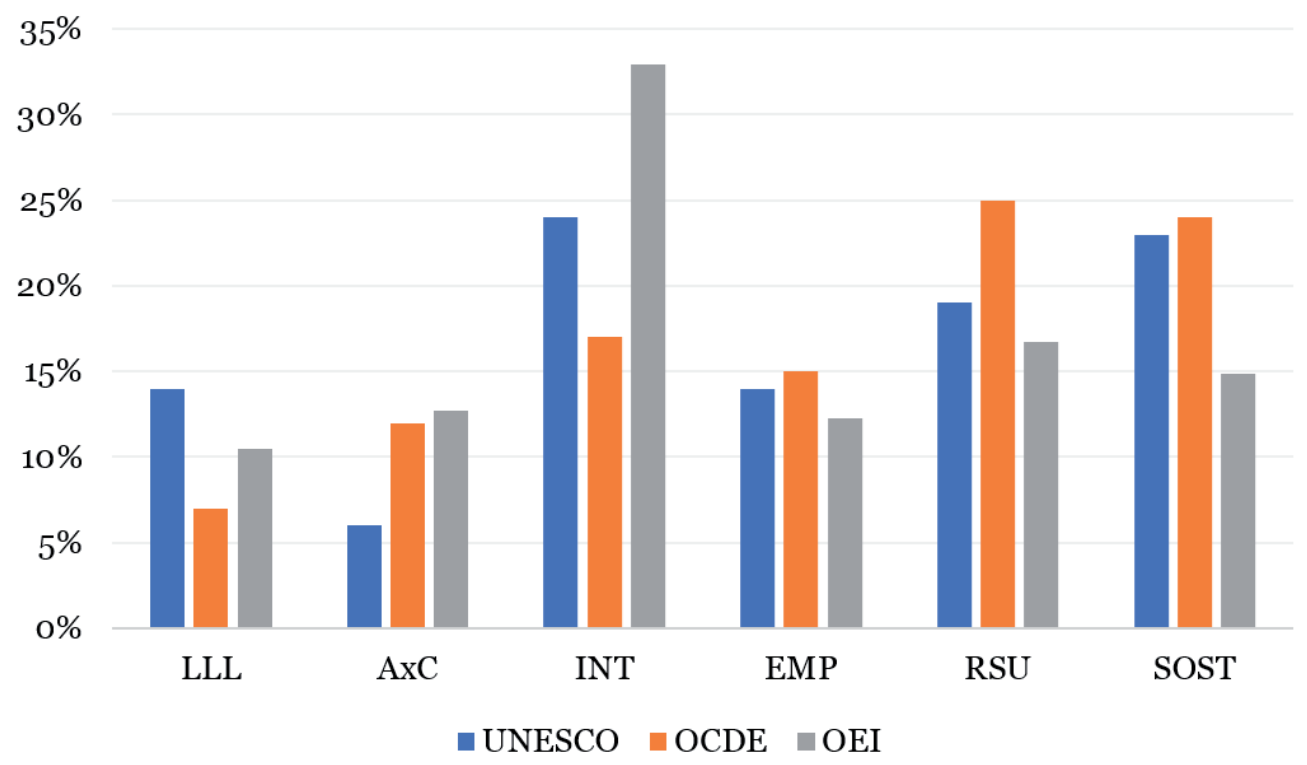

Figura 14. Ámbitos abordados en los documentos UNESCO, OCDE, OEI, periodo 2010-2019

Si bien este estudio no ha utilizado el método comparado, tras el análisis de los documentos seleccionados (2010-2019) para cada uno de los OI (resultando 80 documentos para UNESCO, 26 para OCDE, y 33 para la OEI) se pueden observar algunas diferencias en cuanto a la prevalencia de ciertos ámbitos según organismo (ver Figura 14). Además, se identificaron temáticas para cada uno de los seis ámbitos, encontrando convergencias y divergencias entre ellas y que pasamos a comentar. Para asegurar el aprendizaje a lo largo de la vida, la UNESCO y la OCDE ven fundamental la necesidad de ampliar la oferta de modalidades e itinerarios y, sin embargo, la OEI, considera que el LifelongLearning es fundamental para conservar y promover el derecho a la educación. Por su parte, el aprendizaje por competencias se vincula en la UNESCO y OCDE con las competencias para el siglo XXI, mientras que la OEI asocia las competencias con su propósito educativo y las atiende como un estándar de validación y acreditación.

La apuesta por la internacionalización de la Universidad es compartida por todos los OI, y convergen en sus ejes clave de acción: movilidad estudiantil, armonización de titulaciones y colaboración entre instituciones. Por otro lado, la empleabilidad como objetivo de la educación universitaria se quiere asegurar en la UNESCO y OCDE mediante la promoción de una relación cada vez más estrecha con el mercado laboral y sus necesidades, mientras que la OEI se centra más en la educación técnico-profesional como puente en la educación superior para mejorar la empleabilidad.

Por su parte, la responsabilidad social universitaria queda confirmada como un pilar clave de las nuevas misiones de la educación universitaria. La UNESCO concreta esta responsabilidad en una gobernanza responsable, conocimiento distribuido y responsabilidad financiera, mientras que la OCDE y la OEI hablan de equidad e inclusión. Por último, la sostenibilidad emerge con fuerza en los objetivos universitarios, siendo la gobernanza sostenible un elemento clave y transversal a los tres OI, y enfatizando otros como la sostenibilidad curricular, el desarrollo sostenible y la equidad (UNESCO) y la sostenibilidad financiera (OCDE y OEI).

Los elementos más destacados en cada uno de los OI fueron: reconocimiento de títulos y gobernanza responsable en la UNESCO; armonización de títulos y equidad [RSU] 
en la OCDE; y movilidad y colaboración y cooperación en la OEI. En definitiva, el valor de la internacionalización y la perspectiva de la RSU se muestran, actualmente, como los principales focos de atención de los OI en materia de educación universitaria.

Por lo tanto, los resultados del estudio evidencian la presencia en los documentos de la UNESCO, OCDE y OEI de similitudes en cuanto a la relevancia de los ámbitos indagados y ciertas temáticas recurrentes vinculadas, entre las que se destacan: a) La consideración de la internacionalización universitaria (movilidad, colaboración, conformación de redes) como estrategia para aumentar la calidad de la oferta educativa y el perfilamiento hacia la construcción de universidades globales e instancias masivas de formación online. b) La tendencia hacia una vinculación más estrecha entre la Universidad y el mercado laboral, siendo la empleabilidad uno de los objetivos universitarios actuales. c) Aumento de la responsabilidad de la Universidad con la sociedad, en comparación con sus misiones clásicas. Los nuevos compromisos asignados se vinculan principalmente al desarrollo económico local y la construcción de sociedades más equitativas, proponiendo una mayor apertura institucional con el entorno. d) La sostenibilidad universitaria obedece principalmente a repensar estrategias de gobernanza y de financiamiento públicoprivado que se alinean con la mayor apertura institucional hacia el entorno y la estrecha vinculación con el mercado productivo. e) La preocupación por controlar posibles efectos no deseados fruto de la diversificación y expansión de la oferta universitaria (creación de instituciones de poca calidad, prácticas corruptas, entre otras).

Para concluir, es necesario destacar ciertas limitaciones del estudio presentado. Debido al volumen de información obtenida no ha sido posible proponer distintas miradas interpretativas de los datos. Por este motivo, tal y como se indicaba en la introducción, optamos en esta ocasión por centrarnos en una aproximación fundamentalmente descriptiva, dejando para trabajos posteriores la necesaria problematización de los datos que aquí aportamos. Sin perjuicio de ello, las evidencias recogidas ponen sobre relieve que avanzamos, progresiva pero inexorablemente, hacia un cambio en la misión tradicional de las universidades. Un cambio en las formas y en el fondo, de un profundo calado pedagógico. Sería muy poco deseable que un proceso de transformación de tal relevancia tenga lugar sin reflexiones ni debates que aborden las implicaciones y riesgos que traen consigo los nuevos paradigmas, las nuevas perspectivas y los nuevos valores de la educación superior que hemos explorado aquí. Este artículo aporta evidencias sobre como tres importantísimos OI están abordando la cuestión, poniendo a disposición de la comunidad académica una propuesta de clasificación documental que creemos puede ser de utilidad para continuar indagando sobre el rumbo presente y futuro de la Universidad.

\section{Referencias}

Alba, D. (2017). Hacia una fundamentación de la sostenibilidad en la educación superior. Revista Iberoamericana de Educación, 73(1), 15-34.

Alonso-Sainz, T. (en prensa). Educación para el Desarrollo Sostenible: Una visión crítica desde la Pedagogía. Revista Complutense de Educación.

Alonso-Sainz, T., y Thoilliez, B. (en prensa). Giving Reading, Thinking and Studying Back to Initial Teacher Education Programmes. In Joanna Madalińska-Michalak (Ed.) Studies on Quality Teachers and Quality Teacher Education. Warszawa: Foundation for the Development of the Education System. 
Bárcena, F. (2019). La intimidad del estudio como forma de vida. Teoría de la Educación. Revista Interuniversitaria, 31(2), 41-67.

Bautista-Cerro, M. J. y Díaz, M. J. (2017). La sostenibilidad en los grados universitarios: presencia y coherencia. Teoría de la Educación. Revista Interuniversitaria, 29(1), 161-187.

Carrasco González, A. (2017). Los nuevos modelos de gobernanza universitaria: El caso de la Unión Europea como organismo supranacional que configura el sistema universitario español. Journal of Supranational Policies of Education, Extraordinario, 107-122.

Comisión Europea (2011). Libro Verde. Modernizar la Directiva sobre las cualificaciones profesionales. Recuperado de https://eur-lex.europa.eu/legal-content/ES/TXT/ $\mathrm{PDF} /$ ?uri=CELEX:52011DCo367\&from $=\mathrm{EN}$

Comisión de Rectores de Universidad de España (CRUE) (2012). Directrices para la introducción de la Sostenibilidad en el Curriculum. Recuperado de https:// www.crue.org/Documentos\%20compartidos/Declaraciones/Directrices_ Sosteniblidad_Crue2012.pdf

Delors, J. (1996.). La educación encierra un tesoro. Informe a la UNESCO de la Comisión internacional sobre la educación para el siglo XXI. Madrid: Santillana.

Espejo, L.B. (2006). El aprendizaje permanente y el e-learning. En L. Vega. (Coord.) La sociedad del conocimiento y la educación permanente: retos y riesgos (pp. 6380). Salamanca: Ediciones Universidad de Salamanca

Esteban, F. (2019). La universidad light. Un análisis de nuestra formación universitaria. Barcelona: Paidós.

Eurydice (2001). Iniciativas nacionales para promover el aprendizaje a lo largo de la vida en Europa. Secretaría General de Educación y Formación Profesional (CIDE). Recuperado de https://www.oei.es/historico/etp/iniciativas_nacionales_ promover_aprendizaje_permanente_europa.pdf

Faure, E. (1972). Aprender a ser. La educación del futuro. Recuperado de https://www. berrigasteiz.com/monografikoak/inklusibitatea/pubs/unesco_aprender\%20 a\%20ser.pdf

Fulford, A. (2017). Refusal and disowning knowledge: Re-thinking disengagement in higher education. Ethics and Education, 12(1), 105-115.

Hodgson, N., Vlieghe, J. y Zamojski, P. (Eds.) (2020). Post-critical Perspectives on Higher Education. Reclaiming the Educational in the University. Cham: Springer.

Hodgson, N., Vlieghe, J. y Zamojski, P. (2020). Manifestaciones de lo post-crítico: de principios compartidos a nuevos caminos pedagógicos. Teoría de la Educación. Revista Interuniversitaria, 32(2), 13-23.

Jiménez, J. (2020). Gatherings of Studying: Looking at Contemporary Study Practices in the University. Studies in Philosophy and Education, 39, 269-284. 
Larrosa, J. (2019). Vindicación del estudio como concepto educativo: a propósito de aprender / estudiar una lengua. Teoría de la Educación. Revista Interuniversitaria, 31(2), 131-151.

Manso, J. y Monarca, H. (2016). Concepciones de la OCDE y la Unión Europea sobre el desarrollo profesional docente. Journal of Supranational Policies of Education (JOSPOE), 5, 137-155.

Martínez-Usarralde, M.J., Viana Orta, M.I. y Villarroel, C.B. (2015). La UNESCO Educación en Todos los Sentidos. Valencia: Tirant Lo Blanc.

MECD (2014). Estrategia para la Internacionalización de las Universidades Españolas 2015-2020. Recuperado de https://fe.ccoo.es/ e2e38bo6df949620ed9c4ado4418cc9coooo63.pdf

Medina, B. y Llorent, V. (2017). La educación permanente en los organismos internacionales y las nuevas concepciones. HumanArtes. Revista Electrónica de Ciencias Sociales y Educación, 5(10), 103-121. Recuperado de https://helvia. uco.es/xmlui/bitstream/handle/10396/15024/HumanArtes_Llorent_2017. pdf? sequence $=1$ \&isAllowed $=\mathrm{y}$

Monarca, H. y Manso, J. (2015). Desarrollo profesional docente en el discurso de los organismos internacionales. Revista Española de Educación Comparada, 26, 171-189.

OECD (2015). Adults, Computers and Problem Solving: What's the Problem? OECD Publishing. Recuperado de http://dx.doi.org/10.1787/9789264236844-en

OEI (2010). Metas Educativas 2021: la educación que queremos para la generación de los bicentenarios. Madrid: OEI.

ONU (2015). El camino hacia la dignidad para 2030: acabar con la pobreza y transformar vidas protegiendo el planeta. Recuperado de http://www.hic-gs.org/ content/SGONU_A69700.pdf

ONU (2019). Página principal de la Naciones Unidas. Recuperado de https://www. un.org/sustainabledevelopment/es/objetivos-de-desarrollo-sostenible/

Piñana, E. (2019). La implementación de las competencias clave en Educación Primaria. Un estudio de casos múltiple (Tesis doctoral inédita). Universidad Autónoma de Madrid. Madrid.

Prieto, M. y Manso, J. (2018). La calidad de la educación en los discursos de la OCDE y el Banco Mundial: usos y desusos. En H. Monarca (Coord.) Calidad de la Educación en Iberoamérica: Discursos, políticas y prácticas (pp. 114-135). Madrid: Dykinson.

Quendler, E. y Lamb, M. (2016). Learning as a lifelong process-meeting the challenges of the changing employability landscape: competences, skills and knowledge for sustainable development. International Journal of Continuing Engineering Education and LifeLong Learning, 26(3), 273-293.

Revesado, D. (2018). El proceso de Bolonia y la admisión a la universidad. La realidad del sistema universitario español en perspectiva comparada. Revista Española de Educación Comparada, 32, 169-180. Recuperado de: http://revistas.uned.es/ index.php/REEC/article/view/20948/18710 
Secanella, J. (2016). Nuevas competencias para la empleabilidad de los estudiantes universitarios. La cuestión universitaria. Boletín Electrónico de la Cátedra UNESCO de Gestión y Política Universitaria, 7, 40-47. Recuperado de: http:// polired.upm.es/index.php/lacuestionuniversitaria/article/view/3357/3422

Skea, C. (2017). Student satisfaction in higher education: Settling up and settling down.EthicsandEducation, 12(3),364-377. DOI:10.1080/17449642.2017.1343560

Thoilliez, B. (2014). Las universidades frente a la empleabilidad. Algunos elementos para el análisis. Procedia - Social and Behavioral Sciences, 139, 79-86. DOI: 10.1016/j. sbspro.2014.08.029

Thoilliez, B. (2018). El papel de los organismos internacionales (y de sus expertos, asesores y consultores) en la des-democratización de las políticas educativas. En H. Monarca (Coord.) Calidad de la Educación en Iberoamérica: Discursos, políticas y prácticas (pp. 45-65). Madrid: Dykinson.

Thoilliez, B. y Valle, J. M. (2015). Ser profesor en la Universidad hoy. En F. Gil y D. Reyero (Eds.) Educar en la universidad de hoy: propuestas para la renovación de la vida universitaria (pp. 82-97). Madrid: Encuentro.

Touriñán, J. (2019). Estudiar es actividad común externa y siempre educamos con la actividad. Una aproximación desde la perspectiva mesoaxiológica. Teoría de la Educación. Revista Interuniversitaria, 31(2), 7-39.

UNESCO (2015). Global Report on Adult Learning and Education. Institute for Lifelong Learning: UNESCO. Recuperado de https://www.sel-gipes.com/ uploads/1/2/3/3/12332890/2013_-unesco_-_global_report_on_learning_and_ education_2.pdf

UNESCO (2016). Educación 2030: Declaración de Incheon y Marco de Acción para la realización del Objetivo de Desarrollo Sostenible 4: Garantizar una educación inclusiva y equitativa de calidad y promover oportunidades de aprendizaje permanente para todos. ED-2016/WS/28. Recuperado de https://unesdoc. unesco.org/ark:/48223/pfoooo245656_spa

Valle, J. M. (2015). Las políticas educativas en tiempos de globalización: la educación supranacional. Bordón. Revista de Pedagogía, 67(1), 11-21.

Valle, J.M. y Manso, J. (2013). Competencias clave como tendencia de la política educativa supranacional de la Unión Europea. Revista de Educación, Número Extraordinario, 12-33.

Valle, J.M. y Manso, J. (2017). Lifelong Teacher Education. Ser docente a lo largo de la vida. Madrid: Sial Pigmalión.

Zelaya, M. (2016). Organismos Internacionales y Educación universitaria: Indicios y tensiones. El caso argentino (1990-2000). Journal of Supranational Policies of Education, 4, 121-141. Recuperado de https://revistas.uam.es/index.php/jospoe/ article/view/5669 


\section{ANEXOS}

\section{Anexo 1. Conformación de las muestras de documentos}

\section{A. Conformación de la muestra de documentos para la UNESCO}

Para llevar a cabo la búsqueda documental sobre la producción de documentos en materia de Educación Superior por parte de la UNESCO durante la década sometida a estudio (2010-2019), también acudimos a la librería digital del organismo: UNESCO Digital Library. Para el caso de la UNESCO consistió en dos búsquedas: la primera (Title 'Higher Education', Main topic 'University', From '2010, To '2019', Source 'UNESCO') arrojó un total de 182 registros; en la segunda búsqueda (Main topic 'Higher Education', From '2010', To '2019', Source 'UNESCO') se obtuvieron otros 730 registros; resultando estas dos búsquedas iniciales en un total inicial de 912 documentos. A continuación, pasamos a la fase de cribado donde los resultados obtenidos se exportaron a una hoja de cálculo de Excel eliminando las duplicidades generadas por registros que se presentaban como independientes pero que en realidad eran un mismo documento traducido a diferentes idiomas (dejando en la base de datos solo la versión en inglés o en español), o bien, por repetición entre las dos operaciones de búsqueda ejecutadas en la fase anterior. A continuación, en la fase de elegibilidad se aplicaron los siguientes criterios de exclusión: disponibilidad en línea, no ser documentos de análisis o programa país, no ser memorias de actividades sectoriales, no ser artículos de revistas científicas asociadas a la UNESCO, no ser discursos o cartas de invitación, no ser documentos de creación de centros, no ser informes de viabilidad, no ser informes parciales o versiones ejecutivas de informes más amplios, no ser programas de congresos. Tras la aplicación de estos criterios, el primer grupo de registros se redujo en 114 documentos y el segundo lo hizo en otros 665 documentos, constituyéndose una base de datos con un total de 133 entradas. Por último, en la fase de definición la decisión definitiva sobre qué documentos someteríamos a análisis de contenido, nos llevó a dejar fuera de este un total de 53 documentos, cuyo contenido se circunscribía de manera exclusiva a análisis específicos de regiones. El foco de la investigación es cómo la UNESCO produce discursos políticos en materia de Educación Superior a escala global, no tanto la forma en que escala sus grandes lineamientos estratégicos a las diferentes realidades regionales de Asia, África, América Latina o Europa (regiones sobre las que más documentos región ha producido la UNESCO en la última década). La muestra final que con que trabajamos el análisis de contenido para el caso UNESCO se compuso de un total final de 80 documentos.

\section{B. Conformación de la muestra de documentos para la OCDE}

A partir de la herramienta de búsqueda avanzada de la OECDiLibrary se ha filtrado por temática y años (2010-2019). Se recogieron los datos del número total de cada una de las temáticas principales de la OCDE, esto es: Agriculture and Food; Development; Economics; Education; Employment; Energy; Environment; Finance and Investment; Governance; Industry and Services; Nuclear Energy; Science and Technology; Social Issues/Migration/Health; Taxation; Trade; Transport y Urban, Rural and Regional Development. El número total de publicaciones fue 28521 documentos, de las cuales 3352 pertenecían a la temática de Education, para el período 2010-2019. Seguidamente, para el mismo periodo, filtramos por palabras clave Higher Education y University, dentro 
de la temática de Education, resultando un n=238. Más tarde, se procedió al cribado de los documentos y a desechar material según los siguientes criterios de exclusión: (i) que fuese un informe de un país, (b) que el tipo de documento fuera un gráfico o revista, (c) que el documento no lo firmara la OCDE. Tras este cribado, resultaron 26 documentos.

\section{Conformación de la muestra de documentos para la OEI}

Para analizar la agenda, objetivos y principios propuestos por la OEI en torno a la educación universitaria se ha llevado a cabo un proceso de selección de documentos con ciertas variaciones al seguido para los dos casos anteriores. La disposición de la información en la página web del organismo y la propia naturaleza de la organización han requerido una mayor adaptación de la metodología utilizada para conformar la muestra documental. Para recoger la información buscada en torno a las seis dimensiones de análisis se exploraron dos páginas web: el sitio de la OEI Secretaria Central y la del Observatorio de la Ciencia, Tecnología y Sociedad, instituto creado en el 2008 por la OEI para estudios estratégicos sobre ciencia, tecnología y sociedad y cuenta con el apoyo de red de universidades iberoamericanas. La búsqueda de documentos en la página principal del organismo se realizó a través de las pestañas superiores que organizan la información. De las nueve pestañas se desecharon tres por no estar vinculadas al campo educativo ni a definiciones institucionales. En las seis pestañas restantes se revisaron los correspondientes menús desplegables y se escogieron aquellos que tuviesen documentos descargables e hipervínculos que pudieran llevar a sitios con documentos. A fin de controlar la repetición de sitios indagados y la migración de la página web principal hacia otros sitios, en los casos de presencia de hipervínculos se limitó la búsqueda de documentos a un solo redireccionamiento por enlace. Un tratamiento especial supuso el apartado Biblioteca digital presente en el menú de la pestaña «Desarrollo institucional». En este caso se utilizó la herramienta «búsqueda por autores» ofrecida en el sitio y se recogieron todos los documentos de autoría OEI con fecha de publicación 2010-2019. De este modo, en la página principal de la OEI se revisaron todos los documentos descargables presentes en los apartados de seis menús desplegables (y respectivos redireccionamientos de hasta un nivel) y los documentos de autoría OEI resultantes de la «búsqueda por autor» de la «biblioteca digital» del sitio. Durante este proceso se observó la estrecha relación entre la educación universitaria y el campo científico propuesta por la OEI. Tanto en la página principal como en los documentos explorados, las referencias a la temática universitaria están vinculada o asociadas al desarrollo científico. Por ese motivo, se decidió ampliar la averiguación a la página del Observatorio de Ciencia, Tecnología y Sociedad, instituto especialmente creado por la OEI para el tratamiento de estudios estratégicos sobre ciencia, tecnología y sociedad, que cuenta con el apoyo de red de universidades iberoamericanas. En el sitio web de la página se consideraron todos los documentos descargables con fecha de publicación 2010-2019 de la pestaña Archivo documental. El proceso detallado dio como resultado la identificación de 172 materiales descargables en formato .pdf. A partir de este universo de documentos se conformó la muestra de material de análisis, seleccionando aquellos que cumplían los siguientes requisitos: abordar el tema universitario (de manera exclusiva o parcial) y estar firmado por la OEI, por alguno de expertos responsables o haber sido un estudio elaborado por otro experto, pero solicitado por la organización. Además, se descartaron documentos repetidos en otro idioma y si el contexto al que referían era de un solo país. Esto dio como resultado la muestra final de análisis, compuesta por 33 documentos. 
ANEXO 2. Documentos analizados para el caso UNESCO 2010-2019

\begin{tabular}{|c|c|c|c|c|}
\hline Código & Año & Título & $\begin{array}{l}\text { Tipo de } \\
\text { publicación }\end{array}$ & $\begin{array}{l}\text { Autoría } \\
\text { corporativa }\end{array}$ \\
\hline UNo1 & 2017 & $\begin{array}{l}\text { A paradigm shift in higher education teaching } \\
\text { and learning: practices towards education for } \\
\text { sustainability }\end{array}$ & book part & $\begin{array}{l}\text { UNESCO } \\
\text { (Makrakis, } \\
\text { V.; Kostoulas- } \\
\text { Makrakis, N.) }\end{array}$ \\
\hline UNo2 & 2017 & $\begin{array}{l}\text { Accountability mechanisms in scholarship } \\
\text { awards }\end{array}$ & Book & $\begin{array}{l}\text { UNESCO } \\
\text { (Balfour, S.) }\end{array}$ \\
\hline UNo3 & 2017 & $\begin{array}{l}\text { Accountability of higher education } \\
\text { institutions }\end{array}$ & $\begin{array}{l}\text { programme } \\
\text { and meeting } \\
\text { document }\end{array}$ & $\begin{array}{l}\text { UNESCO } \\
\text { (Semyonov, D.; } \\
\text { Platonova, D.) }\end{array}$ \\
\hline UNo4 & 2017 & $\begin{array}{l}\text { Activities on education, training and research } \\
\text { for sustainable development within the } \\
\text { UNITWIN Network }\end{array}$ & $\begin{array}{l}\text { programme } \\
\text { and meeting } \\
\text { document }\end{array}$ & $\begin{array}{l}\text { UNESCO } \\
\text { (Ricard, M.) }\end{array}$ \\
\hline UNo5 & 2016 & $\begin{array}{l}\text { Advisory statement for effective international } \\
\text { practice: combatting corruption and } \\
\text { enhancing integrity: a contemporary } \\
\text { challenge for the quality and credibility of } \\
\text { higher education }\end{array}$ & Book & IIEEP \\
\hline UNo6 & 2017 & $\begin{array}{l}\text { An Overview of authorization and quality } \\
\text { assurance of higher education institutions }\end{array}$ & $\begin{array}{l}\text { programme } \\
\text { and meeting } \\
\text { document }\end{array}$ & $\begin{array}{l}\text { Global Education } \\
\text { Monitoring } \\
\text { Report Team } \\
\text { (Kinser, K.; Lane, } \\
\text { J. E.) }\end{array}$ \\
\hline UNo7 & 2017 & $\begin{array}{l}\text { Analysis of determinants of a measure of } \\
\text { sustainability literacy }\end{array}$ & Book & $\begin{array}{l}\text { Global Education } \\
\text { Monitoring } \\
\text { Report Team } \\
\text { (Decamps, A.) }\end{array}$ \\
\hline UNo8 & 2017 & $\begin{array}{l}\text { Best practices in the promotion of learning } \\
\text { pathways and linkages between TVET and } \\
\text { higher education }\end{array}$ & Book & $\begin{array}{l}\text { UNESCO } \\
\text { Executive Board } \\
\text { (202nd) }\end{array}$ \\
\hline UNo9 & 2016 & $\begin{array}{l}\text { Closing the gap: opportunities for distance } \\
\text { education to benefit adult learners in higher } \\
\text { education }\end{array}$ & Book & $\begin{array}{l}\text { UILL (Carlsen, } \\
\text { A.; Holmberg, } \\
\text { C.; Neghina, } \\
\text { C.; Owusu- } \\
\text { Boampong, A.) }\end{array}$ \\
\hline UN10 & 2015 & $\begin{array}{l}\text { Comité Jurídico, segundo informe: informe } \\
\text { de síntesis relativo a la aplicación de la } \\
\text { Recomendación sobre la Convalidación de los } \\
\text { Estudios, Títulos y Diplomas de Enseñanza } \\
\text { Superior de } 1993\end{array}$ & book part & $\begin{array}{l}\text { UNESCO General } \\
\text { Conference } \\
\text { (38th) }\end{array}$ \\
\hline UN11 & 2011 & $\begin{array}{l}\text { Consideration of the Draft Programme } \\
\text { and Budget for } 2012-2013(36 \mathrm{C} / 5) \text { and } \\
\text { recommendations of the Executive Board: } \\
\text { strengthening of engineering at UNESCO }\end{array}$ & $\begin{array}{l}\text { programme } \\
\text { and meeting } \\
\text { document }\end{array}$ & $\begin{array}{l}\text { UNESCO } \\
\text { Executive Board } \\
\text { (186th) }\end{array}$ \\
\hline UN12 & 2010 & $\begin{array}{l}\text { Continuing engineering education and } \\
\text { professional development }\end{array}$ & book part & $\begin{array}{l}\text { UNESCO (Jones, } \\
\text { M.) }\end{array}$ \\
\hline UN13 & 2017 & $\begin{array}{l}\text { Cracking the code: girls' education in science, } \\
\text { technology, engineering and mathematics } \\
\text { (STEM) }\end{array}$ & book part & UNESCO \\
\hline UN14 & 2012 & Developing countries in the e-learning era & Book & $\begin{array}{l}\text { IIEEP (Depover, } \\
\text { C.; Orivel, F.) }\end{array}$ \\
\hline UN15 & 2015 & $\begin{array}{l}\text { Draft preliminary report concerning the } \\
\text { preparation of a global convention on the } \\
\text { recognition of higher education qualifications }\end{array}$ & Book & UNESCO \\
\hline
\end{tabular}




\begin{tabular}{|c|c|c|c|c|}
\hline UN16 & 2019 & $\begin{array}{l}\text { Educación inclusiva e interseccional a lo largo } \\
\text { de la vida, para que nadie quede atrás }\end{array}$ & $\begin{array}{l}\text { programme } \\
\text { and meeting } \\
\text { document }\end{array}$ & $\begin{array}{l}\text { UNESCO (Gil, } \\
\text { F. J) }\end{array}$ \\
\hline UN17 & 2018 & $\begin{array}{l}\text { Enseñanza técnica, profesional, terciaria y de } \\
\text { adultos }\end{array}$ & Book & $\begin{array}{l}\text { Global Education } \\
\text { Monitoring } \\
\text { Report Team }\end{array}$ \\
\hline UN18 & 2010 & $\begin{array}{l}\text { Equity and quality assurance: a marriage of } \\
\text { two minds }\end{array}$ & $\begin{array}{l}\text { programme } \\
\text { and meeting } \\
\text { document }\end{array}$ & $\begin{array}{l}\text { IIEEP (Martin, } \\
\text { M.) }\end{array}$ \\
\hline UN19 & 2013 & $\begin{array}{l}\text { Estudio preliminar sobre los aspectos } \\
\text { técnicos y jurídicos relativos a la conveniencia } \\
\text { de contar con un instrumento normativo } \\
\text { mundial sobre el reconocimiento de las } \\
\text { cualificaciones de la educación }\end{array}$ & Book & $\begin{array}{l}\text { UNESCO } \\
\text { (General } \\
\text { Conference, } \\
\text { 37th) }\end{array}$ \\
\hline UN20 & 2016 & $\begin{array}{l}\text { Evaluation of UNESCO's regional conventions } \\
\text { on the recognition of qualifications in higher } \\
\text { education }\end{array}$ & $\begin{array}{l}\text { programme } \\
\text { and meeting } \\
\text { document }\end{array}$ & UNESCO \\
\hline UN21 & 2017 & $\begin{array}{l}\text { Evaluation of UNESCO's work in capacity } \\
\text { building in the basic sciences and engineering }\end{array}$ & $\begin{array}{l}\text { programme } \\
\text { and meeting } \\
\text { document }\end{array}$ & UNESCO \\
\hline UN22 & 2019 & $\begin{array}{l}\text { Exploring STEM competences for the 21st } \\
\text { century }\end{array}$ & $\begin{array}{l}\text { programme } \\
\text { and meeting } \\
\text { document }\end{array}$ & $\begin{array}{l}\text { UNESCO IBE } \\
\text { (Soo Boon, N.) }\end{array}$ \\
\hline UN23 & 2010 & $\begin{array}{l}\text { External quality assurance of tertiary } \\
\text { education in small states: what are the } \\
\text { options }\end{array}$ & Book & $\begin{array}{l}\text { IIEEP (Martin, } \\
\text { M.) }\end{array}$ \\
\hline UN24 & 2010 & $\begin{array}{l}\text { Finding solutions for sustainable financing of } \\
\text { tertiary education in small states }\end{array}$ & $\begin{array}{l}\text { programme } \\
\text { and meeting } \\
\text { document }\end{array}$ & $\begin{array}{l}\text { IIEEP (Tewarie, } \\
\text { B.) }\end{array}$ \\
\hline UN25 & 2011 & $\begin{array}{l}\text { From gender studies to gender IN studies: } \\
\text { case studies on gender-inclusive curriculum } \\
\text { in higher education }\end{array}$ & book part & $\begin{array}{l}\text { UNESCO ECHE } \\
\text { (Grünberg, L.) }\end{array}$ \\
\hline UN26 & 2017 & $\begin{array}{l}\text { From here to there: the UNITWIN/UNESCO } \\
\text { Chairs Programme in the UN Decade of } \\
\text { Education for Sustainable Development and } \\
\text { the Sustainable Development Goals }\end{array}$ & Book & $\begin{array}{l}\text { UNESCO } \\
\text { (Michelsen, G.; } \\
\text { Wells, P. J.) }\end{array}$ \\
\hline UN27 & 2016 & $\begin{array}{l}\text { Futures for higher education and ICT: } \\
\text { changes due to the use of open content }\end{array}$ & $\begin{array}{l}\text { programme } \\
\text { and meeting } \\
\text { document }\end{array}$ & IITE \\
\hline UN28 & 2019 & $\begin{array}{l}\text { Gender-responsive STEM education: } \\
\text { empowering girls and women for the jobs of } \\
\text { today and tomorrow }\end{array}$ & $\begin{array}{l}\text { programme } \\
\text { and meeting } \\
\text { document }\end{array}$ & UNESCO \\
\hline UN29 & 2011 & $\begin{array}{l}\text { Guidelines for open educational resources } \\
\text { (OER) in higher education }\end{array}$ & $\begin{array}{l}\text { programme } \\
\text { and meeting } \\
\text { document }\end{array}$ & UNESCO \\
\hline UN30 & 2017 & $\begin{array}{l}\text { Guidelines on sustainability science in } \\
\text { research and education }\end{array}$ & $\begin{array}{l}\text { programme } \\
\text { and meeting } \\
\text { document }\end{array}$ & UNESCO \\
\hline $\mathrm{UN}_{31}$ & 2018 & Higher Education for Refugees & $\begin{array}{l}\text { programme } \\
\text { and meeting } \\
\text { document }\end{array}$ & $\begin{array}{l}\text { Global Education } \\
\text { Monitoring } \\
\text { Report Team } \\
\text { (Ferede, M. K.) }\end{array}$ \\
\hline UN32 & 2011 & $\begin{array}{l}\text { Higher linguistic education from the } \\
\text { perspective of reforms: new approaches, } \\
\text { prospects, and challenges }\end{array}$ & $\begin{array}{l}\text { programme } \\
\text { and meeting } \\
\text { document }\end{array}$ & $\begin{array}{l}\text { UNESCO ECHE } \\
\text { (Wells, P. J.; } \\
\text { Zolyan, S.) }\end{array}$ \\
\hline
\end{tabular}




\begin{tabular}{|c|c|c|c|c|}
\hline UN33 & 2011 & $\begin{array}{l}\text { ICT in context of student-driven project- } \\
\text { based active learning }\end{array}$ & $\begin{array}{l}\text { programme } \\
\text { and meeting } \\
\text { document }\end{array}$ & $\begin{array}{l}\text { IITE (Koo, B.; } \\
\text { Cha, J.) }\end{array}$ \\
\hline UN34 & 2013 & $\begin{array}{l}\text { Incluir a los jóvenes: retos para la educación } \\
\text { terciaria técnica en América Latina }\end{array}$ & $\begin{array}{l}\text { programme } \\
\text { and meeting } \\
\text { document }\end{array}$ & IIEEP \\
\hline UN35 & 2013 & $\begin{array}{l}\text { Increased autonomy for universities in Asia: } \\
\text { How to make it work }\end{array}$ & Book & IIEEP \\
\hline UN36 & 2015 & $\begin{array}{l}\text { Informe preliminar relativo a la preparación } \\
\text { de una convención mundial sobre el } \\
\text { reconocimiento de las cualificaciones de la } \\
\text { educación superior }\end{array}$ & book part & $\begin{array}{l}\text { UNESCO } \\
\text { Executive Board, } \\
\text { 197th) }\end{array}$ \\
\hline UN37 & 2015 & $\begin{array}{l}\text { Informe preliminar relativo a la preparación } \\
\text { de una convención mundial sobre el } \\
\text { reconocimiento de las cualificaciones de la } \\
\text { educación superior }\end{array}$ & $\begin{array}{l}\text { programme } \\
\text { and meeting } \\
\text { document }\end{array}$ & $\begin{array}{l}\text { UNESCO General } \\
\text { Conference } \\
\text { (38th) }\end{array}$ \\
\hline $\mathrm{UN} 38$ & 2018 & $\begin{array}{l}\text { Internal quality assurance and employability: } \\
\text { how to strengthen the education-employment } \\
\text { linkage }\end{array}$ & Book & $\begin{array}{l}\text { IIEEP (Martin, } \\
\text { M.) }\end{array}$ \\
\hline UN39 & 2018 & $\begin{array}{l}\text { Internal quality assurance and management: } \\
\text { how to close the loop between evidence and } \\
\text { decision-making }\end{array}$ & $\begin{array}{l}\text { programme } \\
\text { and meeting } \\
\text { document }\end{array}$ & IIEEP \\
\hline UN40 & 2017 & $\begin{array}{l}\text { Internal quality assurance: enhancing higher } \\
\text { education quality and graduate employability }\end{array}$ & Book & $\begin{array}{l}\text { IIEEP (Martin, } \\
\text { M.) }\end{array}$ \\
\hline UN41 & 2018 & $\begin{array}{l}\text { International higher education: shifting } \\
\text { mobilities, policy challenges, and new } \\
\text { initiatives }\end{array}$ & $\begin{array}{l}\text { programme } \\
\text { and meeting } \\
\text { document }\end{array}$ & $\begin{array}{l}\text { Global Education } \\
\text { Monitoring } \\
\text { Report Team } \\
\text { (Bhandari, } \\
\text { R.; Robles, C.; } \\
\text { Farrugia, C.) }\end{array}$ \\
\hline UN42 & 2018 & $\begin{array}{l}\text { Linking external and internal quality } \\
\text { assurance }\end{array}$ & $\begin{array}{l}\text { programme } \\
\text { and meeting } \\
\text { document }\end{array}$ & $\begin{array}{l}\text { IIEEP (Martin, } \\
\text { M.) }\end{array}$ \\
\hline UN43 & 2016 & $\begin{array}{l}\text { Monitoring cross-country performance in } \\
\text { equality of access and affordability of tertiary } \\
\text { education: a review of options }\end{array}$ & $\begin{array}{l}\text { programme } \\
\text { and meeting } \\
\text { document }\end{array}$ & $\begin{array}{l}\text { Global Education } \\
\text { Monitoring } \\
\text { Report Team } \\
\text { (Orr, D.) }\end{array}$ \\
\hline UN44 & 2018 & Mobility of students and professionals & book part & $\begin{array}{l}\text { Global Education } \\
\text { Monitoring } \\
\text { Report Team }\end{array}$ \\
\hline UN45 & 2018 & $\begin{array}{l}\text { Pathways of progression: linking technical } \\
\text { and vocational education and training with } \\
\text { post-secondary education }\end{array}$ & $\begin{array}{l}\text { programme } \\
\text { and meeting } \\
\text { document }\end{array}$ & $\begin{array}{l}\text { UNESCO (Field, } \\
\text { S.; Guez, A.) }\end{array}$ \\
\hline UN46 & 2015 & $\begin{array}{l}\text { Preliminary report concerning the } \\
\text { preparation of a global convention on the } \\
\text { recognition of higher education qualifications }\end{array}$ & $\begin{array}{l}\text { programme } \\
\text { and meeting } \\
\text { document }\end{array}$ & $\begin{array}{l}\text { UNESCO } \\
\text { Executive Board } \\
\text { (197th) }\end{array}$ \\
\hline UN47 & 2015 & $\begin{array}{l}\text { Preliminary report concerning the } \\
\text { preparation of a global convention on the } \\
\text { recognition of higher education qualifications }\end{array}$ & book part & $\begin{array}{l}\text { UNESCO General } \\
\text { Conference } \\
\text { (38th) }\end{array}$ \\
\hline $\mathrm{UN} 48$ & 2017 & $\begin{array}{l}\text { Preliminary revision of the } 1974 \text { Regional } \\
\text { Convention on the Recognition of Studies, } \\
\text { Diplomas and Degrees in Higher Education in } \\
\text { Latin America and the Caribbean (1974) }\end{array}$ & $\begin{array}{l}\text { programme } \\
\text { and meeting } \\
\text { document }\end{array}$ & $\begin{array}{l}\text { UNESCO General } \\
\text { Conference } \\
\text { (39th) }\end{array}$ \\
\hline
\end{tabular}




\begin{tabular}{|c|c|c|c|c|}
\hline UN49 & 2013 & $\begin{array}{l}\text { Preliminary study on the technical and } \\
\text { legal aspects relating to the desirability of a } \\
\text { global standard-setting instrument on the } \\
\text { recognition of higher education qualifications }\end{array}$ & $\begin{array}{l}\text { programme } \\
\text { and meeting } \\
\text { document }\end{array}$ & $\begin{array}{l}\text { UNESCO General } \\
\text { Conference } \\
\text { (37th) }\end{array}$ \\
\hline $\mathrm{UN}_{50}$ & 2017 & $\begin{array}{l}\text { Progress report on the preparation of the } \\
\text { Draft Global Convention on the Recognition } \\
\text { of Higher Education Qualifications }\end{array}$ & $\begin{array}{l}\text { programme } \\
\text { and meeting } \\
\text { document }\end{array}$ & $\begin{array}{l}\text { UNESCO } \\
\text { Executive Board } \\
\text { (202nd) }\end{array}$ \\
\hline $\mathrm{UN}_{51}$ & 2017 & $\begin{array}{l}\text { Progress report on the preparation of the } \\
\text { Draft Global Convention on the Recognition } \\
\text { of Higher Education Qualifications }\end{array}$ & Book & $\begin{array}{l}\text { UNESCO General } \\
\text { Conference } \\
\text { (39th) }\end{array}$ \\
\hline $\mathrm{UN}_{52}$ & 2019 & $\begin{array}{l}\text { Progress report on the preparation of the } \\
\text { Draft Global Convention on the Recognition } \\
\text { of Qualifications concerning Higher } \\
\text { Education }\end{array}$ & $\begin{array}{l}\text { programme } \\
\text { and meeting } \\
\text { document }\end{array}$ & $\begin{array}{l}\text { UNESCO General } \\
\text { Conference } \\
\text { (40th) }\end{array}$ \\
\hline UN53 & 2018 & $\begin{array}{l}\text { Proposal for the convening of an international } \\
\text { conference of States for the revision of the } \\
1978 \text { Convention on the Recognition of } \\
\text { Studies, Diplomas and Degrees in Higher } \\
\text { Education in the Arab States }\end{array}$ & book part & $\begin{array}{l}\text { UNESCO } \\
\text { Executive Board } \\
\text { (204th) }\end{array}$ \\
\hline UN54 & 2015 & $\begin{array}{l}\text { Proyecto de informe preliminar relativo a la } \\
\text { preparación de una convención mundial sobre } \\
\text { el reconocimiento de las cualificaciones de la } \\
\text { educación superior }\end{array}$ & $\begin{array}{l}\text { programme } \\
\text { and meeting } \\
\text { document }\end{array}$ & UNESCO \\
\hline UN55 & 2018 & $\begin{array}{l}\text { Quality and employability in higher } \\
\text { education: viewing internal quality assurance } \\
\text { as a lever for change }\end{array}$ & book & $\begin{array}{l}\text { IIEEP (Martin, } \\
\text { M.; Brennan, } \\
\text { J.; Dill, D. D.; } \\
\text { Harvey, L.; } \\
\text { Lemaitre, M.J.) }\end{array}$ \\
\hline $\mathrm{UN}_{5} 6$ & 2013 & Quality assurance in Higher Education & $\begin{array}{l}\text { programme } \\
\text { and meeting } \\
\text { document }\end{array}$ & UNESCO \\
\hline UN57 & 2017 & $\begin{array}{l}\text { Quality management in higher education: } \\
\text { developments and drivers: results from an } \\
\text { international survey }\end{array}$ & $\begin{array}{l}\text { programme } \\
\text { and meeting } \\
\text { document }\end{array}$ & $\begin{array}{l}\text { IIEEP (Martin, } \\
\text { M.; Parikh, S.) }\end{array}$ \\
\hline $\mathrm{UN}_{5} 8$ & 2013 & $\begin{array}{l}\text { Rankings and accountability in higher } \\
\text { education: uses and misuses }\end{array}$ & $\begin{array}{l}\text { programme } \\
\text { and meeting } \\
\text { document }\end{array}$ & $\begin{array}{l}\text { UNESCO } \\
\text { (Marope, P.T.M.; } \\
\text { Wells, Peter J.; } \\
\text { Hazelkorn, E.) }\end{array}$ \\
\hline UN59 & 2017 & $\begin{array}{l}\text { Research, training, cooperation and } \\
\text { information in the framework of higher } \\
\text { education for sustainable development: a } \\
\text { systemic approach }\end{array}$ & Book & $\begin{array}{l}\text { UNESCO (Novo, } \\
\text { M.; Bautista- } \\
\text { Cerro, J.; Murga } \\
\text { Menoyo, M.Á.) }\end{array}$ \\
\hline UN60 & 2015 & $\begin{array}{l}\text { Revised Convention on the Recognition of } \\
\text { Studies, Certificates, Diplomas, Degrees and } \\
\text { Other Academic Qualifications in Higher } \\
\text { Education in African States }\end{array}$ & book part & UNESCO \\
\hline UN61 & 2011 & $\begin{array}{l}\text { Revision of the } 1981 \text { Convention on the } \\
\text { Recognition of Studies, Certificates, } \\
\text { Diplomas, Degrees and other Academic } \\
\text { Qualifications in Higher Education in the } \\
\text { African States }\end{array}$ & $\begin{array}{l}\text { programme } \\
\text { and meeting } \\
\text { document }\end{array}$ & $\begin{array}{l}\text { UNESCO General } \\
\text { Conference } \\
\text { (36th) }\end{array}$ \\
\hline UN62 & 2010 & $\begin{array}{l}\text { Running to stand still: higher education in a } \\
\text { period of global economic crisis }\end{array}$ & book part & $\begin{array}{l}\text { IIEEP (Varghese, } \\
\text { N.V.) }\end{array}$ \\
\hline UN63 & 2016 & $\begin{array}{l}\text { Scholarships for students from developing } \\
\text { countries: establishing a global baseline }\end{array}$ & book part & UNESCO \\
\hline
\end{tabular}




\begin{tabular}{|c|c|c|c|c|}
\hline UN64 & 2019 & $\begin{array}{l}\text { Seoul Statement: The Tokyo Convention: a } \\
\text { New Era for Mobility and Internationalisation } \\
\text { of Higher Education in the Asia-Pacific } \\
\text { through Qualifications Recognition }\end{array}$ & book part & UNESCO \\
\hline UN65 & 2017 & $\begin{array}{l}\text { Six ways to ensure higher education leaves no } \\
\text { one behind }\end{array}$ & $\begin{array}{l}\text { programme } \\
\text { and meeting } \\
\text { document }\end{array}$ & $\begin{array}{l}\text { IIEEP (Makrakis, } \\
\text { V. }\end{array}$ \\
\hline UN66 & 2011 & $\begin{array}{l}\text { Strategies for change towards sustainability in } \\
\text { tertiary education supported by ICT }\end{array}$ & $\begin{array}{l}\text { programme } \\
\text { and meeting } \\
\text { document }\end{array}$ & IITE \\
\hline UN67 & 2017 & $\begin{array}{l}\text { Sustainable development as a guideline for } \\
\text { higher education: an innovative concept for } \\
\text { teaching and learning }\end{array}$ & $\begin{array}{l}\text { programme } \\
\text { and meeting } \\
\text { document }\end{array}$ & $\begin{array}{l}\text { UNESCO } \\
\text { (Michelsen, G.; } \\
\text { Burandt, S.) }\end{array}$ \\
\hline UN68 & 2011 & Teacher education in the global campus & $\begin{array}{l}\text { programme } \\
\text { and meeting } \\
\text { document }\end{array}$ & $\begin{array}{l}\text { IITE (Nikolov, } \\
\text { R.) }\end{array}$ \\
\hline UN69 & 2017 & $\begin{array}{l}\text { Teaching key environmental topics on } \\
\text { sustainable development: implementing } \\
\text { educational project technology }\end{array}$ & $\begin{array}{l}\text { programme } \\
\text { and meeting } \\
\text { document }\end{array}$ & $\begin{array}{l}\text { UNESCO } \\
\text { (Poghosyan, G.; } \\
\text { Gasparyan, A.; } \\
\text { Grigoryan, M.; } \\
\text { Poghosyan, S.) }\end{array}$ \\
\hline UN70 & 2011 & $\begin{array}{l}\text { Tertiary education in small states: planning in } \\
\text { the context of globalization }\end{array}$ & book part & $\begin{array}{l}\text { IIEEP (Martin, } \\
\text { M.; Bray, M.) }\end{array}$ \\
\hline UN71 & 2014 & $\begin{array}{l}\text { The Diversification of post-secondary } \\
\text { education }\end{array}$ & Book & $\begin{array}{l}\text { IIEEP (Varghese, } \\
\text { N.V.) }\end{array}$ \\
\hline UN72 & 2018 & $\begin{array}{l}\text { The Effects of internal quality assurance: } \\
\text { how to make IQA more effective for quality } \\
\text { enhancement }\end{array}$ & $\begin{array}{l}\text { programme } \\
\text { and meeting } \\
\text { document }\end{array}$ & $\begin{array}{l}\text { IIEEP (Martin, } \\
\text { M.) }\end{array}$ \\
\hline UN73 & 2019 & $\begin{array}{l}\text { The ILO/UNESCO Recommendation } \\
\text { concerning the Status of Teachers (1966) and } \\
\text { the UNESCO Recommendation concerning } \\
\text { the Status of Higher-Education Teaching } \\
\text { Personnel (1997) with a revised Foreword and } \\
\text { Users' Guide, 2016 }\end{array}$ & Book & UNESCO \\
\hline UN74 & 2015 & $\begin{array}{l}\text { The role of higher education in promoting } \\
\text { lifelong learning }\end{array}$ & $\begin{array}{l}\text { programme } \\
\text { and meeting } \\
\text { document }\end{array}$ & $\begin{array}{l}\text { UNESCO (Yang } \\
\text { J.; Schneller, C.; } \\
\text { Roche, S.) }\end{array}$ \\
\hline UN75 & 2011 & $\begin{array}{l}\text { Trade in higher education: the role of the } \\
\text { General Agreement on Trade in Services } \\
\text { (GATS) }\end{array}$ & $\begin{array}{l}\text { programme } \\
\text { and meeting } \\
\text { document }\end{array}$ & $\begin{array}{l}\text { IIEEP (Tilak, } \\
\text { Jandhyala B.G.) }\end{array}$ \\
\hline UN76 & 2011 & $\begin{array}{l}\text { Trends in diversification of post-secondary } \\
\text { education }\end{array}$ & Book & IIEEP \\
\hline UN77 & 2016 & $\begin{array}{l}\text { UNESCO's role in the promotion of linkages } \\
\text { between technical and vocational education } \\
\text { and training (TVET) and higher education } \\
\text { qualifications }\end{array}$ & $\begin{array}{l}\text { programme } \\
\text { and meeting } \\
\text { document }\end{array}$ & $\begin{array}{l}\text { UNESCO } \\
\text { Executive Board } \\
\text { (2ooth) }\end{array}$ \\
\hline UN78 & 2013 & UNITWIN/UNESCO Chairs Programme & $\begin{array}{l}\text { programme } \\
\text { and meeting } \\
\text { document }\end{array}$ & UNESCO \\
\hline UN79 & 2015 & Universities: increasingly global players & $\begin{array}{l}\text { programme } \\
\text { and meeting } \\
\text { document }\end{array}$ & $\begin{array}{l}\text { UNESCO } \\
\text { (Aebischer, P.) }\end{array}$ \\
\hline UN80 & 2013 & $\begin{array}{l}\text { Vocationalization of secondary and higher } \\
\text { education: pathways to the world of work }\end{array}$ & Book & $\begin{array}{l}\text { UNESCO } \\
\text { ICTVET } \\
\text { (Maclean, R.; } \\
\text { Pavlova, M.) }\end{array}$ \\
\hline
\end{tabular}


ANEXO 3. Documentos analizados para el caso OCDE 2010-2019

\begin{tabular}{|c|c|c|c|c|}
\hline Código & Año & Título & $\begin{array}{l}\text { Tipo de } \\
\text { publicación }\end{array}$ & Autoría \\
\hline OCo1 & 2019 & $\begin{array}{l}\text { Benchmarking Higher Education System } \\
\text { Performance }\end{array}$ & Book & OECD \\
\hline $\mathrm{OCO} 2$ & 2017 & $\begin{array}{l}\text { Collaboration, alliance, and merger among } \\
\text { higher education institutions }\end{array}$ & Working Paper & Williams, J. \\
\hline $\mathrm{OCO} 3$ & 2015 & $\begin{array}{l}\text { Ensuring Quality in Cross-Border Higher } \\
\text { Education: Implementing the UNESCO/OECD } \\
\text { Guidelines }\end{array}$ & Book & $\begin{array}{l}\text { Vincent- } \\
\text { Lancrin, S.; } \\
\text { Fisher, D.; } \\
\text { Pfotenhauer, S. }\end{array}$ \\
\hline $\mathrm{OCO} 4$ & 2015 & $\begin{array}{l}\text { E-Learning in Higher Education in Latin } \\
\text { America }\end{array}$ & Book & OECD \\
\hline OCo5 & 2015 & $\begin{array}{l}\text { Are Young People Attaining Higher Levels of } \\
\text { Education than their Parents? }\end{array}$ & Working Paper & OECD \\
\hline OCo6 & 2015 & $\begin{array}{l}\text { How do differences in social and cultural } \\
\text { background influence access to higher } \\
\text { education and the completion of studies? }\end{array}$ & Working Paper & OECD \\
\hline OCo7 & 2014 & $\begin{array}{l}\text { Trade Policy in Asia: Higher Education and } \\
\text { Media Services }\end{array}$ & Book & $\begin{array}{l}\text { OECD y Asian } \\
\text { Development } \\
\text { Bank Institute }\end{array}$ \\
\hline OCo8 & 2014 & $\begin{array}{l}\text { At What Age Do University Students Earn } \\
\text { Their First Degree? }\end{array}$ & WorkingPaper & OECD \\
\hline OCo9 & 2014 & $\begin{array}{l}\text { Benchmarking university governance in the } \\
\text { MENA region }\end{array}$ & Article & $\begin{array}{l}\text { Jaramillo, A.; } \\
\text { Zaafrane, H. }\end{array}$ \\
\hline OC10 & 2014 & $\begin{array}{l}\text { Managing the oversight of international } \\
\text { branch campuses in higher education }\end{array}$ & Article & $\begin{array}{l}\text { Kinser, K.; } \\
\text { Lane, J.E. }\end{array}$ \\
\hline OC11 & 2013 & $\begin{array}{l}\text { The Rationale for Higher Education } \\
\text { Investment in Ibero-America }\end{array}$ & Working Paper & Brunner, J.J. \\
\hline OC12 & 2013 & How Are University Students Changing? & WorkingPaper & OECD \\
\hline $\mathrm{OC} 13$ & 2013 & $\begin{array}{l}\text { Talent development as a university mission: } \\
\text { the quadruple helix }\end{array}$ & Article & $\begin{array}{l}\text { Holm-Nielsen, } \\
\text { L.B.; Thorn, K.; } \\
\text { Dørup Olesen, } \\
\text { J.; Huey, T. }\end{array}$ \\
\hline OC14 & 2013 & $\begin{array}{l}\text { Improving the quality of teaching in a state- } \\
\text { owned, regional university }\end{array}$ & Article & $\begin{array}{l}\text { Ballerini, A.A.; } \\
\text { Albarrán, M.I. }\end{array}$ \\
\hline $\mathrm{OC} 15$ & 2013 & $\begin{array}{l}\text { Quality assurance in higher education in } 20 \\
\text { MENA economies }\end{array}$ & Article & El Hassan, K. \\
\hline $\mathrm{OC} 16$ & 2013 & $\begin{array}{l}\text { Reconciling organizational culture and } \\
\text { external quality assurance in higher education }\end{array}$ & Article & Naidoo, D. \\
\hline $\mathrm{OC} 17$ & 2012 & $\begin{array}{l}\text { Guidelines for Quality Provision in Cross- } \\
\text { Border Higher Education: Where Do We } \\
\text { Stand? }\end{array}$ & Working Paper & $\begin{array}{l}\text { Vincent- } \\
\text { Lancrin, S. ; } \\
\text { Pfotenhauer, S. }\end{array}$ \\
\hline OC18 & 2012 & $\begin{array}{l}\text { What Are the Returns on Higher Education for } \\
\text { Individuals and Countries? }\end{array}$ & Working Paper & OECD \\
\hline OC19 & 2012 & $\begin{array}{l}\text { How Are Countries Around the World } \\
\text { Supporting Students in Higher Education? }\end{array}$ & Working Paper & OECD \\
\hline $\mathrm{OC} 2 \mathrm{O}$ & 2012 & $\begin{array}{l}\text { Managing the University Campus: Exploring } \\
\text { Models for the Future and Supporting Today's } \\
\text { Decisions }\end{array}$ & WorkingPaper & den Heijer, A. \\
\hline $\mathrm{OC} 21$ & 2012 & $\begin{array}{l}\text { A survey of international practice in university } \\
\text { admissions testing }\end{array}$ & Article & $\begin{array}{l}\text { Edwards, D.; } \\
\text { Coates, H.; } \\
\text { Friedman, T. }\end{array}$ \\
\hline
\end{tabular}




\begin{tabular}{lclll}
\hline OC22 & 2011 & $\begin{array}{l}\text { The impact of economic crises on American } \\
\text { universities: lessons from the past }\end{array}$ & Article & El-Khawas, E. \\
\hline OC23 & 2011 & $\begin{array}{l}\text { Critical masses for academic research groups } \\
\text { and consequences for higher education } \\
\text { research policy and management }\end{array}$ & Article & $\begin{array}{l}\text { Kenna, R.; } \\
\text { Berche, B. }\end{array}$ \\
\hline OC24 & 2011 & $\begin{array}{l}\text { Assessing the effects of four budget-balancing } \\
\text { strategies in higher education }\end{array}$ & Article & $\begin{array}{l}\text { Hauptman, } \\
\text { A.M.; Nolan, P. }\end{array}$ \\
\hline OC25 & 2010 & $\begin{array}{l}\text { Quality assurance in higher education as a } \\
\text { political process }\end{array}$ & Article & Skolnik, M.L. \\
\hline OC26 & 2010 & $\begin{array}{l}\text { Characteristics of effective and sustainable } \\
\text { teaching development programmes for quality } \\
\text { teaching in higher education }\end{array}$ & Article & Kanuka, H. \\
\hline
\end{tabular}

ANEXO 4. Documentos analizados para el caso OEI 2010-2019

\begin{tabular}{|c|c|c|c|c|}
\hline Código & Año & Título & $\begin{array}{l}\text { Tipo de } \\
\text { publicación }\end{array}$ & Autoría \\
\hline OEo1 & 2010 & $\begin{array}{l}\text { Conferencia Iberoamericana de Ministros de } \\
\text { Educación }\end{array}$ & Declaración & $\begin{array}{l}\text { Ministros de } \\
\text { Educación de } \\
\text { Iberoamérica } \\
\text { y OEI }\end{array}$ \\
\hline OEO2 & 2010 & Metas Educativas 2021 & $\begin{array}{l}\text { Documento } \\
\text { institucional }\end{array}$ & OEI \\
\hline OEO3 & 2010 & $\begin{array}{l}\text { Ciencia, tecnología y universidad en } \\
\text { Iberoamérica }\end{array}$ & Libro digital & $\begin{array}{l}\text { Observatorio } \\
\text { CTS }\end{array}$ \\
\hline OEO4 & 2011 & Informe Miradas 2011 & $\begin{array}{l}\text { Documento } \\
\text { institucional }\end{array}$ & IESME/OEI \\
\hline OEO5 & 2012 & Memorias 2011-2012 & $\begin{array}{l}\text { Documento } \\
\text { institucional }\end{array}$ & OEI \\
\hline OEo6 & 2012 & Informe Mirada 2012 & $\begin{array}{l}\text { Documento } \\
\text { institucional }\end{array}$ & IESME/OEI \\
\hline OEo7 & 2013 & $\begin{array}{l}\text { Conferencia Iberoamericana de Ministros de } \\
\text { Educación }\end{array}$ & Declaración & $\begin{array}{l}\text { Ministros de } \\
\text { Educación de } \\
\text { Iberoamérica } \\
\text { y OEI }\end{array}$ \\
\hline OEo8 & 2014 & $\begin{array}{l}\text { Ciencia, tecnología e innovación para el } \\
\text { desarrollo y la cohesión social }\end{array}$ & Libro digital & $\begin{array}{l}\text { Observatorio } \\
\text { CTS }\end{array}$ \\
\hline OEO9 & 2014 & Base Convocatoria Paulo Freire & $\begin{array}{l}\text { Documento } \\
\text { institucional }\end{array}$ & OEI \\
\hline OE10 & 2014 & $\begin{array}{l}\text { Conferencia Iberoamericana de Ministros de } \\
\text { Educación }\end{array}$ & Declaración & $\begin{array}{l}\text { Ministros de } \\
\text { Educación de } \\
\text { Iberoamérica } \\
\text { y OEI }\end{array}$ \\
\hline OE11 & 2014 & $\begin{array}{l}\text { Declaración de Puebla Reunión de Ministros } \\
\text { y Altas Autoridades de Ciencia, Tecnología e } \\
\text { Innovación de Iberoamérica }\end{array}$ & Declaración & $\begin{array}{l}\text { Ministros de } \\
\text { Educación de } \\
\text { Iberoamérica } \\
\text { y OEI }\end{array}$ \\
\hline OE12 & 2014 & Memorias 2013-2014 & $\begin{array}{l}\text { Documento } \\
\text { institucional }\end{array}$ & $\begin{array}{l}\text { Ministros de } \\
\text { Educación de } \\
\text { Iberoamérica } \\
\text { y OEI }\end{array}$ \\
\hline OE13 & 2014 & Informe Miradas 2014 & $\begin{array}{l}\text { Documento } \\
\text { institucional }\end{array}$ & IESME/OEI \\
\hline OE14 & 2015 & $\begin{array}{l}\text { VI Reunión del Consejo Rector del Instituto de } \\
\text { Evaluación y Seguimiento de las Metas }\end{array}$ & Acta de reunión & $\begin{array}{l}\text { Consejo Asesor } \\
\text { IESME }\end{array}$ \\
\hline
\end{tabular}




\begin{tabular}{|c|c|c|c|c|}
\hline OE15 & 2015 & Informe Miradas 2015 & $\begin{array}{l}\text { Documento } \\
\text { institucional }\end{array}$ & IESME/OEI \\
\hline OE16 & 2016 & Memorias 2014-2015 & $\begin{array}{l}\text { Documento } \\
\text { institucional }\end{array}$ & OEI \\
\hline OE17 & 2016 & $\begin{array}{l}\text { El sistema iberoamericano. La cooperación al } \\
\text { servicio de la comunidad }\end{array}$ & $\begin{array}{l}\text { Documento } \\
\text { institucional }\end{array}$ & OEI/SEGIB \\
\hline OE18 & 2016 & $\begin{array}{l}\text { Conferencia Iberoamericana de Ministros de } \\
\text { Educación }\end{array}$ & Declaración & $\begin{array}{l}\text { Ministros de } \\
\text { Educación de } \\
\text { Iberoamérica } \\
\text { y OEI }\end{array}$ \\
\hline OE19 & 2016 & Informe Miradas 2016 & $\begin{array}{l}\text { Documento } \\
\text { institucional }\end{array}$ & IESME/OEI \\
\hline OE2O & 2017 & Memorias 2016 & $\begin{array}{l}\text { Documento } \\
\text { institucional }\end{array}$ & OEI \\
\hline OE21 & 2018 & Memorias 2015-2016 & $\begin{array}{l}\text { Documento } \\
\text { institucional }\end{array}$ & OEI \\
\hline OE22 & 2018 & Memoria 2017 & $\begin{array}{l}\text { Documento } \\
\text { institucional }\end{array}$ & OEI \\
\hline OE23 & 2018 & $\begin{array}{l}\text { Informe de coyuntura } \mathrm{N}^{\circ} \text { : Aumenta el } \\
\text { número de estudiantes de educación superior } \\
\text { en Iberoamérica }\end{array}$ & $\begin{array}{l}\text { Documento } \\
\text { institucional }\end{array}$ & $\begin{array}{l}\text { Observatorio } \\
\text { CTS }\end{array}$ \\
\hline OE24 & 2018 & $\begin{array}{l}\text { Las universidades, pilares de la ciencia y de la } \\
\text { tecnología en América Latina }\end{array}$ & Libro digital & $\begin{array}{l}\text { Observatorio } \\
\text { CTS }\end{array}$ \\
\hline OE25 & 2018 & $\begin{array}{l}\text { Conferencia Iberoamericana de Ministros de } \\
\text { Educación }\end{array}$ & Declaración & $\begin{array}{l}\text { Ministros de } \\
\text { Educación de } \\
\text { Iberoamérica } \\
\text { y OEI }\end{array}$ \\
\hline OE26 & 2018 & Informe Miradas 2018 & $\begin{array}{l}\text { Documento } \\
\text { institucional }\end{array}$ & IESME/OEI \\
\hline OE27 & 2019 & $\begin{array}{l}\text { Informe de coyuntura No6: Se expande } \\
\text { la educación superior a distancia en } \\
\text { Iberoamérica, aunque es todavía incipiente }\end{array}$ & $\begin{array}{l}\text { Documento } \\
\text { institucional }\end{array}$ & $\begin{array}{l}\text { Observatorio } \\
\text { CTS }\end{array}$ \\
\hline OE28 & 2019 & $\begin{array}{l}\text { Papel } \mathrm{N}^{\circ} \text { 12: Panorama de la educación } \\
\text { superior en Iberoamérica -Caracterización de } \\
\text { educación superior y acreditación universitaria } \\
\text { Edición } 2019\end{array}$ & $\begin{array}{l}\text { Documento } \\
\text { institucional }\end{array}$ & $\begin{array}{l}\text { Observatorio } \\
\text { CTS }\end{array}$ \\
\hline OE28 & 2019 & $\begin{array}{l}\text { Papel } \mathrm{N}^{\mathrm{o}} \text { 12: Panorama de la educación } \\
\text { superior en Iberoamérica -Caracterización de } \\
\text { educación superior y acreditación universitaria } \\
\text { Edición } 2019\end{array}$ & $\begin{array}{l}\text { Documento } \\
\text { institucional }\end{array}$ & $\begin{array}{l}\text { García de } \\
\text { Fanelli, A. (a } \\
\text { pedido del } \\
\text { Observatorio } \\
\text { CTS) }\end{array}$ \\
\hline OE29 & 2019 & Informe de Evaluación Paulo Freire & $\begin{array}{l}\text { Documento } \\
\text { institucional }\end{array}$ & $\begin{array}{l}\text { Rubio } \\
\text { Armendáriz, C. (a } \\
\text { pedido de la OEI) }\end{array}$ \\
\hline OE30 & 2019 & $\begin{array}{l}\text { Informe Diagnóstico de la Educación Superior } \\
\text { en Iberoamérica }\end{array}$ & $\begin{array}{l}\text { Documento } \\
\text { institucional }\end{array}$ & $\begin{array}{l}\text { Sainz González, } \\
\text { J. (a pedido } \\
\text { de la OEI } \\
\text { (Presentación } \\
\text { de Jabonero) }\end{array}$ \\
\hline OE31 & 2019 & Documento base PIME & $\begin{array}{l}\text { Documento } \\
\text { institucional }\end{array}$ & OEI \\
\hline OE32 & 2019 & $\begin{array}{l}\text { Papel No 10: Panorama de la educación } \\
\text { superior en Iberoamérica a través de los } \\
\text { indicadores de la Red Î́ndices }\end{array}$ & $\begin{array}{l}\text { Documento } \\
\text { institucional }\end{array}$ & $\begin{array}{l}\text { García de Fanelli, } \\
\text { A. (a pedido del } \\
\text { Observatoria } \\
\text { CTS) }\end{array}$ \\
\hline OE33 & 2019 & $\begin{array}{l}\text { Papel } N^{0} \text { 11: Los investigadores universitarios } \\
\text { y su vínculo con el entorno en América Latina }\end{array}$ & $\begin{array}{l}\text { Documento } \\
\text { institucional }\end{array}$ & $\begin{array}{l}\text { Observatorio } \\
\text { CTS }\end{array}$ \\
\hline
\end{tabular}

